\title{
Concentration function of additive functions on shifted twin primes
}

\author{
by \\ Simon Wong (Portales, N.Mex.)
}

0. Introduction. The content of this paper is part of the author's Ph.D. thesis. The two new theorems in this paper provide upper bounds on the concentration function of additive functions evaluated on shifted $\gamma$-twin prime, where $\gamma$ is any positive even integers. Both results are generalizations of theorems due to I. Z. Ruzsa, N. M. Timofeev, and P. D. T. A. Elliott.

Acknowledgements. I would like to thank Professor P. D. T. A. Elliott for his patience and guidance through every stage of this work and Professor I. Z. Ruzsa for his advice in improving this paper.

1. Notation and history. This section merely includes the notation necessary to state our main theorems. All other notation is defined where it first appears.

A real-valued function $f$ is said to be additive if $f(m n)=f(m)+f(n)$ for all $m$ and $n$ that are coprime. Throughout this paper, $f$ will denote an additive function, and $g$ the multiplicative function defined by $g(n)=e^{i t f(n)}$, unless otherwise stated. Moreover, $p$ and $q$ will denote prime numbers while $x$ and $y$ will be real numbers with $y$ greater than 8 . The variable $w$ will denote a sufficiently large power of $\log x$. Throughout the paper, we shall impose finitely many conditions on $w$. We choose $w$ to be the largest power of $\log x$ necessary to satisfy all the conditions. Also, for any fixed nonzero integer $a$, any positive even integer $\gamma<x$, and any real number $h$, define

$$
\begin{gathered}
\Omega_{\gamma}(x)=\{p \leq x: p \text { and } p+\gamma \text { are primes }\} \\
\Psi_{\gamma}(x)=2 \prod_{p>2}\left(1-\frac{1}{(p-1)^{2}}\right) \prod_{2<p \mid \gamma}\left(\frac{p-1}{p-2}\right) \frac{x}{\log ^{2} x}, \\
Q_{h}(x, \gamma)=\frac{1}{\Psi_{\gamma}(x)}\left|\left\{p \in \Omega_{\gamma}(x): h<f(p+a) \leq h+1\right\}\right| .
\end{gathered}
$$

1991 Mathematics Subject Classification: Primary 11N13. 
The absolute value denotes the cardinality of the enclosed set. Since $\gamma$ remains fixed for a majority of the paper, we shall generally suppress the dependence of $Q_{h}$ on $x$ and $\gamma$.

The function $\sup _{h} Q_{h}$ is nearly a concentration function of the additive function $f$ evaluated on shifted $\gamma$-twin primes. It would, in fact, be a concentration function if the number of $\gamma$-twin primes grew asymptotically as $\Psi_{\gamma}(x)$. The last statement, of course, is unproven.

Concentration functions of additive functions have been the object of much research in number theory. Ruzsa studied the concentration function on integers:

$$
\sup _{h} \widetilde{Q}_{h}(x)=\sup _{h} \frac{1}{x}|\{n \leq x: h<f(n) \leq h+1\}| .
$$

Let

$$
W(x)=\min _{\lambda \in \mathbb{R}}\left(\lambda^{2}+\sum_{p \leq x} \frac{1}{p} \min (1,|f(p)-\lambda \log p|)^{2}\right) .
$$

In 1980, Ruzsa [7] showed that $\sup _{h} \widetilde{Q}_{h}(x)$, the concentration function on integers, is $\ll W(x)^{-1 / 2}$. Timofeev [8] conducted the pioneering work on the concentration function of shifted primes in the early 1990s. He obtained a result similar to, but weaker than, Ruzsa's result. Then in 1992, Elliott [4] improved on Timofeev's ideas and proved an analog to Ruzsa's result for concentration function on shifted primes. The definition of $\widetilde{Q}_{h}$ is adjusted in the obvious way in the shifted primes case.

If $\pi_{\gamma}(x)$ denotes the cardinality of $\Omega_{\gamma}(x)$, then A. F. Lavrik proved that the difference between $\Psi_{\gamma}(x)$ and $\pi_{\gamma}(x)$ is small in an averaged sense over $\gamma$.

Theorem 0 (Lavrik). Let $\Lambda(n)$ be the von Mangoldt function. Then for any $c>0$,

$$
\begin{array}{r}
\sum_{0<\gamma \leq x}\left|\sum_{\substack{0<m, n \leq x \\
m-n=\gamma}} \Lambda(m) \Lambda(n)-2 \prod_{p>2}\left(1-\frac{1}{(p-1)^{2}}\right) \prod_{\substack{p \mid \gamma \\
p>2}}\left(\frac{p-1}{p-2}\right)(x-\gamma)\right| \\
\ll x^{2}(\log x)^{-c} .
\end{array}
$$

Proof. This is Lemma 17.3 of [6].

Observe that

$$
\begin{aligned}
\sum_{0<\gamma \leq x} \sum_{\substack{0<m, n \leq x+\gamma \\
m-n=\gamma}} \frac{\Lambda(m) \Lambda(n)}{\log ^{2} x} & =\sum_{0<\gamma \leq x} \sum_{\substack{p \leq x \\
q=p+\gamma}} \frac{\log p \log q}{\log ^{2} x}+O\left(x^{3 / 2}\right) \\
& \leq 2 \sum_{0<\gamma \leq x} \pi_{\gamma}(x)+O\left(x^{3 / 2}\right) .
\end{aligned}
$$


Lavrik's theorem thus implies that

$$
\sum_{0<\gamma \leq x} \pi_{\gamma}(x)+O\left(\frac{x^{2}}{\log ^{c} x}\right) \geq \frac{1}{2} \sum_{0<\gamma \leq x} \Psi_{\gamma}(x) .
$$

If $\gamma$ were an even integer for which $\pi_{\gamma}(x) \sim \Psi_{\gamma}(x)$ as $x \rightarrow \infty$, then $Q_{h}(x, \gamma)$ would be precisely the concentration function of the shifted $\gamma$-twin primes. Provided such a $\gamma$ exists, Theorem 1 below shows that the concentration function $Q_{h}$ is $\ll(1+W(x))^{-1 / 2}$. We remove the proviso in Theorem 2 by showing that the concentration functions are small in an averaged sense over $\gamma$.

\section{Statement of results}

THEOREM 1. Let $y$ be an absolute constant not less than $8, w$ be a sufficiently large fixed power of $\log x$, and $w<z \leq x^{1 / 10}$. Then for any additive function $f$,

$$
Q_{h}=Q_{h}(x, \gamma) \ll \prod_{p \mid a(\gamma-a)}\left(1+\frac{2}{p}\right) \frac{1}{\sqrt{1+W(x)}},
$$

and the bound holds uniformly for all $h, x, \gamma$, and $f$.

Furthermore, the functions $Q_{h}$ satisfy a similar bound on average over $\gamma$.

TheOREM 2. In the notation of Theorem 1 , let $\pi_{\gamma}(x)$ be the cardinality of the set $\Omega_{\gamma}(x)$. Then

$$
\left(\sum_{\gamma \leq x} \pi_{\gamma}(x)\right)^{-1} \sum_{\gamma \leq x} \Psi_{\gamma}(x) \sup _{h} Q_{h}(x, \gamma) \ll \frac{1}{\sqrt{1+W(x)}}
$$

for some absolute constant $c$. The implied constant depends at most on a.

3. Preliminary lemmas. Before embarking on the proof of Theorem 1, we shall require the assistance of the following lemmas.

Lemma 1. Let $g(n)$ be a multiplicative arithmetic function with modulus not greater than one. Let $x$ and $T$ be real numbers greater than two. Define

$$
m(T)=m(x, T)=\min _{|t| \leq T} \sum_{p \leq x} p^{-1}\left(1-\operatorname{Re}\left(\frac{g(p)}{p^{i t}}\right)\right) .
$$

For any squarefree integer with $(D, a(a-\gamma))=1$, we have

$$
\sum_{n \leq x} g(n) \prod_{\substack{p|D, p| n \\ p \nmid \gamma}}\left(\frac{p-1}{p-3}\right) \prod_{\substack{p|D, p| n \\ p \mid \gamma}}\left(\frac{p-1}{p-2}\right) \ll x\left(\exp \left(-\frac{m(T)}{8}\right)+T^{-1 / 4}\right) .
$$

Proof. Lemma 1 is essentially Lemma 6.10 in Elliott's text [2] on probabilistic number theory. Lemma 1 is a generalization of a result of G. Halász. 
The difference in our setting is the presence of the extraneous product terms. This means the multiplicative function in our summand may no longer be bounded by one in absolute value.

The proof of Lemma 6.10 of [2] continues to hold even in our setting. Having an upper bound of one on the summand is not absolutely necessary. However, it is vital that when $n$ is a prime greater than $y$,

$$
\prod_{p \mid n}\left(\frac{p-1}{p-2}\right) \leq \prod_{p \mid n}\left(\frac{p-1}{p-3}\right) \leq 2 .
$$

To further streamline notation, we shall use $P_{\gamma}(n)$ to denote the polynomial $(n-a)(n-a+\gamma)$, and let $\varrho_{\gamma}(k)$ be the number of solutions to $P_{\gamma}(n) \equiv 0$ $(\bmod k)$ in $\mathbb{Z} / k \mathbb{Z}$, for any positive integer $k$. Thus, for any $p \nmid \gamma, \varrho_{\gamma}(p)=2$, for $p \mid \gamma, \varrho_{\gamma}(p)=1$. Finally, for any squarefree integer $k, \varrho_{\gamma}(k) \leq 2^{\omega(k)}$, where $\omega(k)$ counts the number of distinct prime divisors of $k$. Let

$$
P_{m, n}=\prod_{\substack{m<p \leq n \\ p \nmid a, p \nmid(a-\gamma)}} p .
$$

Lemma 2 (Selberg). Suppose $\lambda_{d}$ are real numbers with $\lambda_{1}=1, w<z$, and $P$ is a product of distinct primes. Define a multiplicative function $g$ by

$$
g(d)= \begin{cases}\frac{\varrho_{\gamma}(d)}{d} \prod_{p \mid d}\left(1-\frac{\varrho_{\gamma}(p)}{p}\right)^{-1} & \text { for } \mu(d) \neq 0 \\ 0 & \text { otherwise. }\end{cases}
$$

Also, let

$$
\mathfrak{G}_{k}(x)=\sum_{\substack{d<x \\(d, k)=1}} \mu^{2}(d) g(d) .
$$

Then the quadratic form

$$
\sum_{\substack{d_{j} \mid P \\ j=1,2}} \lambda_{d_{1}} \lambda_{d_{2}} \frac{\varrho_{\gamma}\left(\left[d_{1}, d_{2}\right]\right)}{\left[d_{1}, d_{2}\right]} \quad \text { with } \lambda_{d_{j}}=0 \text { for } d_{j} \geq z
$$

is minimized by choosing

$$
\lambda_{d}=\mu(d) \prod_{p \mid d}\left(1-\frac{\varrho_{\gamma}(p)}{p}\right)^{-1} \frac{\mathfrak{G}_{d}(z / d)}{\mathfrak{G}_{1}(z)},
$$

and the minimal value is $\mathfrak{G}_{1}^{-1}(z)$. Furthermore, for any $\lambda>0$,

$\mathfrak{G}_{1}^{-1}(z)=\prod_{p \mid P}\left(1-\frac{\varrho_{\gamma}(p)}{p}\right)\left(1+O\left\{\exp \left(-\lambda \frac{\log x}{\log z}+\left(\frac{4}{\lambda}+\frac{4}{\log z} e^{\lambda}\right)\right)\right\}\right)$

if $z \leq x$. 
The constant $P$ above will often be $P_{w, z}$, with $z \leq x^{1 / 10}$, in our applications of this lemma. In that case,

$$
\frac{1}{\mathfrak{G}_{1}(z)} \ll \Pi_{1}\left(\frac{\log w}{\log z}\right)^{2},
$$

where

$$
\Pi_{1}=\prod_{\substack{p\left|P_{w}, z \\ p\right| \gamma}}\left(\frac{p-1}{p-2}\right) \prod_{\substack{w<p \leq z \\ p \mid a(a-\gamma)}}\left(\frac{p}{p-2}\right) .
$$

Pro of (of Lemma 2). This lemma is a combination of equations (3.1.4), (3.1.7), and Lemma 4.1 of Halberstam and Richert [5]. In fact, Halberstam and Richert prove a much more general result than the one stated here. Note that for $\gamma<x$ and $w>\log x$,

$$
\Pi_{1} \ll \log \log x .
$$

Lemma 3. Let $B \geq 0$. There is a constant $c$ so that if $2 \leq Q \leq M$, then those pairs $(\chi, \tau)$ of Dirichlet characters to moduli not exceeding $Q$, and of reals $\tau,|\tau| \leq Q^{B}$, for which

$$
\sum_{Q<p \leq M} \frac{1}{p}\left(1-\operatorname{Re}\left(g(p) \chi(p) p^{i \tau}\right)\right)<\frac{1}{4} \log \left(\frac{\log M}{\log Q}\right)-c
$$

have the property that the characters are all induced by the same primitive character. For the purposes of this statement all principal characters are regarded as induced by the function which is identically one on all positive integers.

Proof. See the proofs of Lemmas 3 and 4 of [3].

Lemma 4. Let $0<\beta<1,0<\varepsilon<1 / 8$, and $2 \leq \log M \leq Q \leq M$. Then

$$
\sum_{\substack{n \leq x \\ n \equiv r(\bmod D)}} g(n)=\frac{1}{\phi(D)} \sum_{\substack{n \leq x \\(n, D)=1}} g(n)+O\left(\frac{x}{\phi(D)}\left(\frac{\log Q}{\log x}\right)^{1 / 8-\varepsilon}\right)
$$

uniformly for $M^{\beta} \leq x \leq M$, all $r$ prime to $D$, and all moduli $D$ not exceeding $Q$ save possibly for the multiples of a single modulus $D_{0}>1$.

Proof. This is part of Lemma 10 of [4].

Lemma 5. In the notation of Lemma 3 , let $0<\beta<1,0<\varepsilon<1 / 8$, $\Delta \mid P_{y, w}$, and $2 \leq Q \leq \min \left(M, x^{1 / 4}\right)$. Moreover, let $g$ be any multiplicative function with modulus bounded by one. Then 


$$
\begin{aligned}
& \sum_{\substack{n \leq x \\
n \equiv r(\bmod D)}} g(n) \prod_{\substack{p|\Delta \\
p| n, p \nmid \gamma}}\left(\frac{p-1}{p-3}\right) \prod_{\substack{p|\Delta \\
p| n, p \mid \gamma}}\left(\frac{p-1}{p-2}\right) \\
&-\frac{1}{\phi(D)} \sum_{\substack{n \leq x \\
(n, D)=1}} g(n) \prod_{\substack{p|\Delta \\
p| n, p \nmid \gamma}}\left(\frac{p-1}{p-3}\right) \prod_{\substack{p|\Delta \\
p| n, p \mid \gamma}}\left(\frac{p-1}{p-2}\right) \\
& \ll \frac{x}{\phi(D)}\left(\frac{\log Q}{\log x}\right)^{1 / 8-\varepsilon}
\end{aligned}
$$

uniformly for $M^{\beta} \leq x \leq M$, all $r$ prime to $D$, and all moduli $D$ not exceeding $Q$ save possibly for the multiples of a single modulus $D_{0}>1$.

The modulus $D_{0}$ in Lemma 5 is the modulus to which the primitive character in Lemma 3 is defined.

To facilitate notation, for any positive integers $m, n, l$, let

$$
G_{l}(m, \gamma, n)=\prod_{\substack{p|m, p \nmid \gamma \\ p| n, p \nmid l}}\left(\frac{p-1}{p-3}\right) \prod_{\substack{p|m, p| \gamma \\ p \mid n, p \nmid l}}\left(\frac{p-1}{p-2}\right) .
$$

The dependence on $\gamma$ will be suppressed in the situations where there is no possible confusion. Further, when $l=1$, we shall write $G(m, n)$ instead of $G_{1}(m, n)$.

Pro of (of Lemma 5). For any fixed positive integer $l$, define a multiplicative function $v$ by the Dirichlet convolution

$$
G_{l}(\Delta, n)=\sum_{d \mid n} v(d) \quad \text { and } \quad v(p)=0 \quad \text { for } p \nmid \Delta \text { or } p \mid l .
$$

For $p \mid \Delta$ and $p \nmid l$,

$$
v\left(p^{k}\right)= \begin{cases}\frac{2}{p-3} & \text { if } k=1 \text { and } p \nmid \gamma \\ \frac{1}{p-2} & \text { if } k=1 \text { and } p \mid \gamma, \\ 0 & \text { if } k>1 .\end{cases}
$$

For any prime $p$, we have $2 /(p-3)>1 /(p-2)$; thus, for any $\alpha>0$,

$$
\sum_{d=1}^{\infty} \frac{v(d)}{d^{\alpha}} \leq \prod_{p}\left(1+\frac{2}{p^{\alpha}(p-3)}\right) \ll 1 .
$$

To prove Lemma 5, first suppose that $g$ is completely multiplicative. 
Then

$$
\begin{aligned}
& \sum_{\substack{n \leq x \\
n \equiv r(D)}} g(n) G_{l}(\Delta, n)-\frac{1}{\phi(D)} \sum_{\substack{n \leq x \\
(n, D)=1}} g(n) G_{l}(\Delta, n) \\
& =\sum_{\substack{d \leq x \\
(d, \bar{D})=1}} v(d) g(d)\left(\sum_{\substack{m \leq x / d \\
m \equiv r d^{-1}(D)}} g(m)-\frac{1}{\phi(D)} \sum_{\substack{m \leq x / d \\
(m, D)=1}} g(m)\right),
\end{aligned}
$$

since $(r, D)=1$. Divide the outer sum into two, one with $d \leq \sqrt{x}$ and the other with $\sqrt{x}<d \leq x$. For $d \leq \sqrt{x}$, Lemma 4 gives an upper bound of

$$
\ll \sum_{\substack{d \leq \sqrt{x} \\(d, D)=1}} \frac{v(d) x}{d \phi(D)}\left(\frac{\log Q}{\log (x / d)}\right)^{1 / 8-\varepsilon} \ll \frac{x}{\phi(D)}\left(\frac{\log Q}{\log x}\right)^{1 / 8-\varepsilon} .
$$

For $\sqrt{x}<d \leq x$, we estimate directly. According to inequality (4), this portion of (5) contributes

$$
\ll \sum_{\substack{\sqrt{x}<d \leq x \\(d, D)=1}} v(d)\left(\frac{x}{d D}+1+\frac{x}{d \phi(D)}\right) \ll \frac{x^{3 / 4}}{\phi(D)} .
$$

Consequently, (5) is bounded by

$$
\frac{x}{\phi(D)}\left(\frac{\log Q}{\log x}\right)^{1 / 8-\varepsilon}
$$

Clearly, this bound holds uniformly in $l$. By selecting $l=1$, the lemma is proved in the case when $g$ is completely multiplicative.

To remove the requirement of complete multiplicativity, express $g$ as a convolution of $g_{1}$ and $g_{2}$, where $g_{1}$ is completely multiplicative and is defined by $g_{1}\left(p^{k}\right)=g(p)^{k}$. Then $g_{2}(p)=0$, and one verifies by induction on $k$ that

$$
g_{2}\left(p^{k}\right)=g\left(p^{k}\right)-g(p) g\left(p^{k-1}\right),
$$

for $k \geq 2$. Hence, $\left|g_{2}\left(p^{k}\right)\right| \leq 2$ for $k \geq 2$. It follows immediately that, for any $\alpha>0$ and $l=1$,

(6) $\quad \sum_{n \leq x} \frac{\left|g_{2}(n)\right|}{n^{\alpha}} G(\Delta, n) \ll \sum_{d \leq x} \frac{v(d)}{d^{\alpha}} \prod_{p \leq x / d}\left(1+\frac{2}{p^{2 \alpha}}+\frac{2}{p^{3 \alpha}}+\ldots\right)$.

So, the sum is $\ll 1$ for $\alpha=1$ and is $\ll \log ^{2} x$ for $\alpha=1 / 2$.

With these definitions for $g_{1}$ and $g_{2}$, we see that for $(r, D)=1$,

$$
\sum_{\substack{n \leq x \\ n \equiv r(D)}} g(n) G(\Delta, n)=\sum_{\substack{d \leq x \\(d, D)=1}} g_{2}(d) G(\Delta, d) \sum_{\substack{m \leq x / d \\ m \equiv r d^{-1}(D)}} g_{1}(m) G_{d}(\Delta, m) .
$$


Similarly,

$$
\sum_{\substack{n \leq x \\(n, D)=1}} g(n) G(\Delta, n)=\sum_{\substack{d \leq x \\(d, D)=1}} g_{2}(d) G(\Delta, d) \sum_{\substack{m \leq x / d \\(m, D)=1}} g_{1}(m) G_{d}(\Delta, m) .
$$

In view of these two remarks, the difference we wish to estimate in the lemma is

$$
\sum_{\substack{d \leq x \\(d, \bar{D})=1}} g_{2}(d) G(\Delta, d) F(x, d, D)
$$

where

$$
F(x, d, D)=\sum_{\substack{m \leq x / d \\ m \equiv r d^{-1}(D)}} g_{1}(m) G_{d}(\Delta, m)-\frac{1}{\phi(D)} \sum_{\substack{m \leq x / d \\(m, D)=1}} g_{1}(m) G_{d}(\Delta, m) .
$$

Since $g_{1}$ is completely multiplicative, $F(x, d, D)$ is identical to the left-hand side of (5) with $l=d$. We employ a similar tactic as before by dividing the outer sum in two: $d \leq x^{3 / 4}$ and $x^{3 / 4}<d \leq x$.

For $d \leq x^{3 / 4}$, the result already obtained for completely multiplicative functions shows that this portion of $(7)$ is

$$
\ll\left(\frac{\log Q}{\log x}\right)^{1 / 8-\varepsilon} \frac{x}{\phi(D)} \sum_{\substack{d \leq x^{3 / 4} \\(d, D)=1}} \frac{\left|g_{2}(d)\right|}{d} G(\Delta, d) .
$$

From the remarks following inequality (6), this bound is no greater than

$$
\frac{x}{\phi(D)}\left(\frac{\log Q}{\log x}\right)^{1 / 8-\varepsilon} \text {. }
$$

As for $x^{3 / 4}<d \leq x$, note that

$$
\prod_{p \mid \Delta}\left(\frac{(p-1)^{2}}{(p-3)(p-2)}\right) \ll \log ^{3} w
$$

because $\Delta \mid P_{y, w}$ implies $\Delta \ll e^{w}$. Therefore, this portion of (7) is dominated by

$$
x \log ^{3} w x^{-3 / 8} \sum_{x^{3 / 4}<d \leq x} \frac{\left|g_{2}(d)\right|}{\sqrt{d}} G(\Delta, d) .
$$

Estimate (6) shows that the last expression is

$$
\ll x^{5 / 8}(\log \log x)^{7} \log ^{2} x \ll x^{3 / 4} \ll \frac{x}{\phi(D)}\left(\frac{\log Q}{\log x}\right)^{1 / 8-\varepsilon},
$$

as $\phi(D) \leq Q \leq x^{1 / 4}$. This concludes the proof of Lemma 5 . 
For any positive integer $m$, define

$$
H(m, \gamma)=\prod_{\substack{p \mid m \\ p \nmid \gamma}}\left(\frac{p-3}{p-1}\right) \prod_{\substack{p|m \\ p| \gamma}}\left(\frac{p-2}{p-1}\right) .
$$

As before, the dependence on $\gamma$ will be suppressed when there is no possible confusion.

Lemma 6 . Let $2 \leq r \leq x$. Then the number of integers not exceeding $x$ and made up entirely of primes not exceeding $r$ is

$$
\ll x \exp \left(-12 \frac{\log x}{\log r}\right)+x^{14 / 15} .
$$

Proof. This is Lemma 13 of [3].

Lemma 7. Let $y>e$, and $w$ be a fixed power of $\log x$. Also, let $G\left(P_{y, w}, \gamma, n\right), D_{0}$, and $Q$ be as defined in Lemma 5 . As before, for $1 \leq j \leq$ $\varrho_{\gamma}\left(D_{0}\right), b_{j}$ will denote the solutions of the congruence $P_{\gamma}(n) \equiv 0\left(\bmod D_{0}\right)$. Then either

$$
\sum_{\substack{n \leq x \\\left(P_{\gamma}(n), P_{y, w}\right)=1}} g(n)=H\left(P_{y, w}\right) \sum_{n \leq x} g(n) G\left(P_{y, w}, n\right)+O\left(x(\log x)^{-1 / 20}\right),
$$

or

$$
\begin{aligned}
\sum_{\substack{n \leq x \\
\left(P_{\gamma}(n), P_{y, w}\right)=1}} g(n)= & H\left(P_{y, w}\right) \sum_{n \leq x} g(n) G\left(P_{y, w}, n\right) \\
& +H\left(P_{y, w}\right) c_{\gamma}\left(D_{0}\right) \\
& \times \sum_{j=1}^{\varrho_{\gamma}\left(D_{0}\right)} \bar{\chi}\left(b_{j}\right) \sum_{n \leq x} \chi(n) g(n) G\left(\frac{P_{y, w}}{D_{0}}, n\right) \\
& +O\left(x(\log x)^{-21 / 20}\right),
\end{aligned}
$$

where

$$
c_{\gamma}\left(D_{0}\right)=\frac{\mu\left(D_{0}\right)}{D_{0}} \prod_{\substack{p \mid D_{0} \\ p \nmid \gamma}}\left(\frac{p}{p-3}\right) \prod_{\substack{p\left|D_{0} \\ p\right| \gamma}}\left(\frac{p}{p-2}\right)
$$

and $\chi$ is a primitive Dirichlet character modulo $D_{0}$. Moreover, $D_{0}$ divides $P_{y, w}, D_{0} \leq Q$, and $\omega\left(D_{0}\right) \leq \frac{1}{25} \log \log x$.

Pr o of. Set $M=x$ and $Q=\exp \left((\log \log x)^{3}\right)$ in Lemma 5. First, suppose there are no exceptional moduli relative to $g$. As a consequence of Brun's 
pure sieve, for any positive integers $n$ and $r$,

$$
\sum_{d \mid n} \mu(d)=\sum_{\substack{d \mid n \\ \omega(d) \leq r-1}} \mu(d)+\alpha \sum_{\substack{d \mid n \\ \omega(d)=r}} \mu(d),
$$

where $0 \leq \alpha \leq 1$. This is Corollary 2.24 of [5].

The relatively prime condition $\left(P_{\gamma}(n), P_{y, w}\right)=1$ may be replaced by using the Möbius function. Then the sum we wish to estimate equals

$$
\begin{gathered}
\sum_{\substack{d \mid P_{y, w} \\
d \leq Q \\
\omega(d) \leq r-1}} \mu(d) \sum_{\substack{n \leq x \\
P_{\gamma}(n) \equiv 0(d)}} g(n)+\sum_{\substack{d \mid P_{y, w} \\
d \leq Q \\
\omega(d)=r}} \mu(d) \sum_{\substack{n \leq x \\
P_{\gamma}(n) \equiv 0(d)}} \alpha g(n) \\
+\sum_{\substack{d \mid P_{y, w} \\
Q<d \leq P_{\gamma}(x) \\
\omega(d) \leq r-1}} \mu(d) \sum_{\begin{array}{c}
n \leq x \\
P_{\gamma}(n) \equiv 0(d)
\end{array}} g(n)+\sum_{\substack{d \mid P_{y, w} \\
Q<d \leq P_{\gamma}(x) \\
\omega(d)=r}} \mu(d) \sum_{\substack{n \leq x \\
P_{\gamma}(n) \equiv 0(d)}} \alpha g(n) .
\end{gathered}
$$

The value of $\alpha$ may depend upon $y, w, n, \gamma$, and the polynomial $P_{\gamma}(n)$. However, since $\alpha$ is bounded between 0 and 1 , it causes no difficulties upon taking its absolute value.

Set $r=(\log \log x)^{3}$. With this choice of $r, d \leq Q$ and $d \mid P_{y, w}$ imply $\omega(d)<r$. Consequently, in the first term of (8), the restriction on $\omega(d)$ may be omitted. Moreover, if $d \mid P_{y, w}$, and $\omega(d)=r$, then $d>Q$; and so, the second term is void. As for the last two terms, in the range of $d$, an upper bound of $\sqrt{x}$ may be used in place of $P_{\gamma}(x)$. Then the last two terms of (8) are

$$
\begin{aligned}
& \ll x \sum_{\substack{d \mid P_{y, w} \\
Q<d \leq \sqrt{x}}} \frac{\mu^{2}(d) \varrho_{\gamma}(d)}{d}+\sum_{\substack{d \mid P_{y, w} \\
Q<d \leq \sqrt{x}}} \mu^{2}(d) \varrho_{\gamma}(d) \\
& \ll x\left(\sum_{d \mid P_{y, w}} \frac{4^{\omega(d)}}{d}\right)^{1 / 2}\left(\sum_{\substack{d \mid P_{y, w} \\
Q<d \leq \sqrt{x}}} \frac{1}{d}\right)^{1 / 2} .
\end{aligned}
$$

It is easy to see that the first product is $\ll \log ^{2} w$. In the second product, divide the interval $(Q, \sqrt{x}]$ into disjoint intervals of the form $\left(2^{k} U, 2^{k+1} U\right]$, where $U=Q$ and $k=0,1,2, \ldots$ Lemma 6 provides a bound on the number of integers not exceeding $x$ which consist only of primes up to $w$. Applying this lemma on each subinterval, we get

$$
\sum_{\substack{d \mid P_{y, w} \\ U<d \leq 2^{k+1} U}} \frac{1}{d} \leq \frac{1}{2^{k} U} \sum_{\substack{d \mid P_{y, w} \\ 2^{k} U<d \leq 2^{k+1} U}} 1 \ll \exp \left(-\frac{\log U}{\log w}\right)+U^{-1 / 15} .
$$


As it requires $\ll \log x$ of such subintervals to cover $(Q, \sqrt{x}]$,

$$
\left(\sum_{\substack{d \mid P_{y, w} \\ Q<d \leq \sqrt{x}}} \frac{1}{d}\right)^{1 / 2} \ll\left(\log x \exp \left(-\frac{\log Q}{\log w}\right)\right)^{1 / 2} \ll \exp \left(-(\log \log x)^{3 / 2}\right) .
$$

Hence, the last two terms of (8) are less than the error term of Lemma 7. The main contribution arises from the first double sum of (8).

Since there are no exceptional moduli by assumption, the first term of

(8) may be estimated by $\varrho_{\gamma}(d)$ applications of Lemma 4 . It equals

(9) $\sum_{\substack{d \mid P_{y, w} \\ d \leq Q}} \frac{\mu(d) \varrho_{\gamma}(d)}{\phi(d)} \sum_{\substack{n \leq x \\(n, d)=1}} g(n)+O\left(\sum_{\substack{d \mid P_{y, w} \\ d \leq Q}} \frac{x \mu^{2}(d) \varrho_{\gamma}(d)}{\phi(d)}\left(\frac{\log Q}{\log x}\right)^{1 / 8-\varepsilon}\right)$.

By our choice of $Q$, the quantity in the error term is

$$
\ll x\left(\frac{(\log \log x)^{3}}{\log x}\right)^{1 / 8-\varepsilon} \prod_{y<p \leq w}\left(1+\frac{2}{p-1}\right) \ll \frac{x}{(\log x)^{1 / 10}} .
$$

Removing the condition $d \leq Q$ from the main term in (9) introduces an error that is

$$
\ll x \exp \left(-(\log \log x)^{3 / 2}\right) .
$$

This estimate may be obtained by appealing to Lemma 6 as before.

Therefore, the main term of (9) equals

$$
\sum_{n \leq x} g(n) \sum_{\substack{d \mid P_{y, w} \\(d, n)=1}} \frac{\mu(d) \varrho_{\gamma}(d)}{\phi(d)}+O\left(x \exp \left(-(\log \log x)^{3 / 2}\right)\right)
$$

Since

$$
\sum_{\substack{d \mid P_{y, w} \\(d, n)=1}} \frac{\mu(d) \varrho_{\gamma}(d)}{\phi(d)}=G\left(P_{y, w}, n\right) H\left(P_{y, w}\right)
$$

Lemma 7 is proved in the case where there are no exceptional moduli.

Now suppose there are exceptional moduli. According to Lemma 5, all exceptional moduli are multiples of a single modulus, $1<D_{0} \leq Q$. The only place where the above argument may fail is in estimating the first term of (8) with Lemma 4. The bound used may not be valid for the exceptional moduli.

If $D_{0}$ does not divide $P_{y, w}$, then there would be no difficulties because $d \mid P_{y, w}$ and thus $d$ is not a multiple of $D_{0}$. Moreover, if $D_{0}>Q$, then no $d \leq Q$ can be a multiple of $D_{0}$. Hence, if $D_{0} \nmid P_{y, w}$ or if $D_{0}>Q$, the first estimate of Lemma 7 is again valid. Finally, if $\omega\left(D_{0}\right)>\frac{1}{25} \log \log x$ and 
$D_{0} \mid P_{y, w}$, then

$$
\frac{\varrho_{\gamma}\left(D_{0}\right)}{D_{0}} \leq\left(\frac{2}{11}\right)^{\omega\left(D_{0}\right)} \leq(\log x)^{-1 / 19}
$$

Therefore, the middle term in the second estimate of Lemma 7 is

$$
\ll \frac{x(\log \log \log x)^{5}}{(\log x)^{1 / 19}} \ll x(\log x)^{-1 / 20} .
$$

Once again, the first estimate of Lemma 7 holds in this setting. Without loss of generality, suppose $1<D_{0} \leq Q, D_{0} \mid P_{y, w}$, and $\omega\left(D_{0}\right) \leq \frac{1}{25} \log \log x$ for the remainder of the proof.

For any $d \mid P_{y, w}$, define $d_{1}=\left(d, D_{0}\right)$ and $d_{2}=d / d_{1}$. Since $d$ is squarefree, we have $\left(d_{1}, d_{2}\right)=1$ and $\left(d_{2}, D_{0}\right)=1$. Furthermore, any Dirichlet character with squarefree modulus $d$ may be factorized into a product of characters, $\chi_{1} \chi_{2}$, where $\chi_{1}$ has modulus $d_{1}$ and $\chi_{2}$ has modulus $d_{2}$. This simple observation will permit us to divide any character into two parts, with the modulus of one dividing $D_{0}$ and the modulus of the other being coprime to $D_{0}$.

Proceeding as in the case where there were no exceptional moduli, we get

$$
\begin{aligned}
\sum_{\substack{n \leq x \\
\left(P_{\gamma}(n), P_{y, w}\right)=1}} g(n)= & \sum_{\substack{d \mid P_{y, w} \\
d \leq Q}} \mu(d) \sum_{\substack{n \leq x \\
P_{\gamma}(n) \equiv 0(d)}} g(n) \\
& +O\left(x \exp \left(-(\log \log x)^{3 / 2}\right)\right) .
\end{aligned}
$$

As a consequence of the Chinese Remainder Theorem, the main term on the right-hand side equals

$$
\sum_{\substack{d \mid P_{y}, w \\ d \leq Q}} \mu(d) \sum_{\substack{n \leq x \\ P_{\gamma}(n) \equiv 0\left(d_{1}\right) \\ P_{\gamma}(n) \equiv 0\left(d_{2}\right)}} g(n) .
$$

For $m=1$ or 2 , let $c_{m l}, 1 \leq l \leq \varrho_{\gamma}\left(d_{m}\right)$, be the solutions to $P_{\gamma}(n) \equiv 0$ $\left(\bmod d_{m}\right)$. Then, with the aid of the orthogonality properties of characters, (10) may be rewritten as

$$
\sum_{\substack{d \mid P_{y, w} \\ d \leq Q}} \frac{\mu(d)}{\phi\left(d_{1}\right)} \sum_{j=1}^{\varrho_{\gamma}\left(d_{1}\right)} \sum_{\chi_{1}} \bar{\chi}_{1}\left(c_{1 j}\right) \sum_{\substack{n \leq x \\ P_{\gamma}(n) \equiv 0\left(d_{2}\right)}} g(n) \chi_{1}(n) .
$$

In view of Lemma 3, inequality (2) only holds for characters induced by a common primitive character $\chi\left(\bmod D_{0}\right)$. Suppose $\psi$ is a character modulo $d_{2}$. If $\chi_{1}$ is not a character induced by $\chi$, then for $\psi$ being principal, $\chi_{1} \psi$ would not be induced by $\chi$ either. On the other hand, if $\chi_{1}$ and $\chi_{1} \psi$ are both characters induced by $\chi$, then for any integer $n$ with $\left(n, d_{1} d_{2}\right)=1$ and $n \equiv 1\left(\bmod D_{0}\right)$, 


$$
1=\chi_{1} \psi(n)=\chi_{1}(n) \psi(n)=\psi(n) .
$$

The above identity implies $\psi$ must be a principal character because $D_{0} \nmid d_{2}$. Hence, if $\chi_{1}$ is induced by $\chi$, then for any nonprincipal $\psi, \chi_{1} \psi$ cannot be induced by the character $\chi$.

In either case, for any $\chi_{1}$, there exists a $\psi\left(\bmod d_{2}\right)$ such that $g \chi_{1} \psi$ cannot satisfy inequality (2) of Lemma 3 . We are, therefore, free to apply Lemma 4 to estimate the sum over $g \chi_{1}$ in (11). Thus the sum of the $g(n)$ 's that we wish to estimate in Lemma 7 equals

$$
\begin{aligned}
& \sum_{\substack{d \mid P_{y, w} \\
d \leq Q}} \frac{\mu(d)}{\phi\left(d_{1}\right)} \sum_{j=1}^{\varrho_{\gamma}\left(d_{1}\right)} \sum_{\chi_{1}} \bar{\chi}_{1}\left(c_{1 j}\right) \frac{\varrho_{\gamma}\left(d_{2}\right)}{\phi\left(d_{2}\right)} \sum_{\substack{n \leq x \\
\left(n, d_{2}\right)=1}} g(n) \chi_{1}(n) \\
& +O\left(\left|\sum_{\substack{d \mid P_{y, w} \\
d \leq Q}} \frac{\mu(d)}{\phi\left(d_{1}\right)} \sum_{j=1}^{\varrho_{\gamma}\left(d_{1}\right)} \sum_{\chi_{1}}\right| \bar{\chi}_{1}\left(c_{1 j}\right)\left|\frac{x \varrho_{\gamma}\left(d_{2}\right)}{\phi\left(d_{2}\right)}\left(\frac{\log Q}{\log x}\right)^{1 / 8-\varepsilon}\right|\right) .
\end{aligned}
$$

The error term in (12) is

$$
\ll \frac{x}{(\log x)^{1 / 20}} .
$$

As for the main terms in (12), switching the order of summation and using, again, the orthogonality of characters gives

$$
\sum_{j=1}^{\varrho_{\gamma}\left(d_{1}\right)} \sum_{\substack{\left.n \leq x \\ n, d_{2}\right)=1 \\ n \equiv c_{1 j}\left(d_{1}\right)}} g(n)=\sum_{\substack{n \leq x \\\left(n, d_{2}\right)=1 \\ P_{\gamma}(n) \equiv 0\left(d_{1}\right)}} g(n) .
$$

As in the setting where there were no exceptional moduli, we remove the condition that $d$ does not exceed $Q$. This introduces an error which is

$$
\ll x(\log \log \log x)^{2} \exp \left(-(\log \log x)^{3 / 2}\right) .
$$

Thus the error from eliminating the restriction on the magnitude of $d$ may be absorbed by the error term in Lemma 7 .

It is convenient to write $P_{0}$ for $P_{y, w} / D_{0}$. Consequently, it follows from (12) that

$$
\begin{aligned}
\sum_{\substack{n \leq x \\
\left(P_{\gamma}(n), P_{y, w}\right)=1}} g(n)= & \sum_{n \leq x} g(n) \sum_{\substack{d_{1}\left|D_{0} \\
d_{1}\right| P_{\gamma}(n)}} \mu\left(d_{1}\right) \sum_{\substack{d_{2} \mid P_{0} \\
\left(d_{2}, n\right)=1}} \frac{\mu\left(d_{2}\right) \varrho_{\gamma}\left(d_{2}\right)}{\phi\left(d_{2}\right)} \\
& +O\left(\frac{x}{(\log x)^{1 / 20}}\right) .
\end{aligned}
$$


Converting the sum over $d_{2}$ into its product form, the main term becomes

$$
H\left(P_{0}\right) \sum_{m \mid D_{0}} \mu(m) \sum_{\substack{n \leq x \\ P_{\gamma}(n) \equiv 0(m)}} g(n) G\left(P_{0}, n\right) .
$$

Let $b_{m j}, 1 \leq j \leq \varrho_{\gamma}(m)$, be the solutions to $P_{\gamma}(n) \equiv 0(\bmod m)$. Since

$$
\sum_{\substack{n \leq x \\ P_{\gamma}(n) \equiv 0(m)}} g(n) G\left(P_{0}, n\right)=\sum_{j=1}^{\varrho_{\gamma}(m)} \sum_{\substack{n \leq x \\ n \equiv b_{m j}(m)}} g(n) G\left(P_{0}, n\right),
$$

we may apply Lemma 5 to the inner sum over $n$ when $m<D_{0}$. Expression (13) has a representation

$$
\begin{aligned}
& H\left(P_{0}\right) \sum_{\substack{m \mid D_{0} \\
m<D_{0}}} \frac{\mu(m)}{\phi(m)} \sum_{j=1}^{\varrho_{\gamma}(m)} \sum_{\substack{n \leq x \\
(n, m)=1}} g(n) G\left(P_{0}, n\right) \\
&+H\left(P_{0}\right) \mu\left(D_{0}\right) \sum_{j=1}^{\varrho_{\gamma}\left(D_{0}\right)} \sum_{\substack{n \leq x \\
n \equiv b_{D_{0}, j}\left(D_{0}\right)}} g(n) G\left(P_{0}, n\right) \\
&+ O\left(H\left(P_{0}\right) \frac{x}{(\log x)^{1 / 10}}\right) .
\end{aligned}
$$

Add the term

$$
\frac{\mu\left(D_{0}\right) \varrho_{\gamma}\left(D_{0}\right)}{\phi\left(D_{0}\right)} \sum_{\substack{n \leq x \\\left(n, D_{0}\right)=1}} g(n) G\left(P_{0}, n\right)
$$

to the first term of (14) and subtract the same from the second. The first term of (14) plus (15) equals

$$
H\left(P_{0}\right) \sum_{n \leq x} g(n) G\left(P_{0}, n\right) \sum_{\substack{m \mid D_{0} \\(m, n)=1}} \frac{\mu(m) \varrho_{\gamma}(m)}{\phi(m)} .
$$

The inner sum over $m$ equals $G\left(D_{0}, n\right) H\left(D_{0}\right)$. Since $P_{0}=P_{y, w} / D_{0}$, we get

$$
G\left(P_{0}, n\right) G\left(D_{0}, n\right)=G\left(P_{y, w}, n\right) \quad \text { and } \quad H\left(P_{0}\right) H\left(D_{0}\right)=H\left(P_{y, w}\right) .
$$

So (16) yields the first term in the second estimate of Lemma 7.

The second term in (14) may be expressed as

$$
H\left(P_{0}\right) \frac{\mu\left(D_{0}\right)}{\phi\left(D_{0}\right)} \sum_{j=1}^{\varrho_{\gamma}\left(D_{0}\right)} \sum_{\chi_{0}\left(\bmod D_{0}\right)} \bar{\chi}_{0}\left(b_{D_{0}, j}\right) \sum_{n \leq x} g(n) \chi_{0}(n) G\left(P_{0}, n\right) .
$$


The last expression is the same as (15) when $\chi_{0}$ is the principal character modulo $D_{0}$. Thus the difference between the second term of (14) and expression (15) is

$$
H\left(P_{0}\right) \frac{\mu\left(D_{0}\right)}{\phi\left(D_{0}\right)} \sum_{j=1}^{\varrho_{\gamma}\left(D_{0}\right)} \sum_{\chi_{0}} \bar{\chi}_{0}\left(b_{j}\right) \sum_{\substack{n \leq x \\\left(n, D_{0}\right)=1}} g(n) \chi_{0}(n) G\left(P_{0}, n\right),
$$

where the middle sum is over all characters modulo $D_{0}$ which are nonprincipal. To obtain the statement of the lemma, use the identity

$$
H\left(P_{0}\right) \frac{\mu\left(D_{0}\right)}{\phi\left(D_{0}\right)}=H\left(P_{y, w}\right) c_{\gamma}\left(D_{0}\right)
$$

When $\chi_{0}$ is the exceptional primitive character, $\chi,(17)$ is precisely the middle term of Lemma 7's second estimate. When $\chi_{0} \neq \chi$, the inequality in (2) points in the opposite direction because then these characters cannot be induced by $\chi$. Lemma 1 thus yields

$$
\sum_{n \leq x} \chi_{0}(n) g(n) G\left(P_{0}, n\right) \ll x(\log x)^{-1 / 9},
$$

provided $y>8$. Consequently, for $\chi_{0} \neq \chi$ and $\chi_{0}$ being nonprincipal, (17) is

$$
\ll \frac{x}{(\log x)^{1 / 9}} H\left(P_{0}\right) \frac{\left|\mu\left(D_{0}\right) \varrho_{\gamma}\left(D_{0}\right)\right|}{\phi\left(D_{0}\right)} \sum_{\substack{\chi_{0} \neq \chi \\ \text { nonprincipal }}} 1 .
$$

Because $\omega\left(D_{0}\right) \leq \frac{1}{25} \log \log x$, the last expression is $\ll x(\log x)^{-1 / 20}$. Lemma 7 is established.

4. Proof of Theorem 1. Armed with the above lemmas, we proceed to prove Theorem 1. For any real $u$,

$$
\int_{-1}^{1}(1-|t|) e^{i t u} d t=2 \int_{0}^{1}(1-t) \cos (t u) d t=\left(\frac{\sin (u / 2)}{u / 2}\right)^{2} .
$$

Hence, the integral is nonnegative for all real $u$. Moreover,

$$
\left(\frac{\sin (u / 2)}{u / 2}\right)^{2} \geq \frac{1}{2} \quad \text { for }|u| \leq 1 .
$$

Thus when $|f(n+a)-h| \leq 1$ and $g=e^{i t f}$,

$$
2 \int_{-1}^{1}(1-|t|) e^{-i t h} g(n+a) d t \geq 1 .
$$

Since $8 \leq y \leq z<x$, the set of primes $p$ in $(z, x]$ such that $p \nmid a(a-\gamma)$, $p+\gamma$ is also prime, and $h<f(p+a) \leq h+1$ is a subset of the set of the 
integers $n$ with $\left(n(n+\gamma), P_{y, z}\right)=1$ which satisfy $h<f(n+a) \leq h+1$.

Thus,

$$
Q_{h} \leq \frac{2}{\Psi_{\gamma}(x)} \sum_{\substack{n \leq x \\\left(n(n+\gamma), P_{y, z}\right)=1}} \int_{-1}^{1}(1-|t|) e^{-i t h} g(n+a) d t+\frac{z}{\Psi_{\gamma}(x)}+o(1) .
$$

We introduce real numbers $\lambda_{d},\left|\lambda_{d}\right| \leq 1,1 \leq d \leq z$, with $\lambda_{1}=1$, reminiscent of Selberg's sieve method. Because the integral in (18) is nonnegative, for $y \leq w \leq z, Q_{h}$ is not more than a constant multiple of

$$
\int_{-1}^{1} \frac{(1-|t|) e^{-i t h}}{\Psi_{\gamma}(x)} \sum_{\substack{d_{j} \leq z \\ d_{j} P_{w, z} \\ j=1,2}} \lambda_{d_{1}} \lambda_{d_{2}} \sum_{\substack{n \leq x \\\left(n(n+\gamma), P_{y, w}=1 \\ n(n+\gamma) \equiv 0\left(\left[d_{1}, d_{2}\right]\right)\right.}} g(n+a) d t+\frac{z}{\Psi_{\gamma}(x)} .
$$

In the sum involving the $\lambda$ 's in (19), when it is clear that $j$ varies from 1 to 2 , the condition $j=1,2$ will be omitted.

Following the ideas in Elliott's paper [4], define the following functions:

$$
\begin{aligned}
g_{1}(p)= & g(p), \quad g_{1}\left(p^{k}\right)=\frac{g(p)^{k}}{k !}, \quad g_{1}(p q)=g_{1}(p) g_{1}(q), \\
g(n)= & h * g_{1}(n), \\
\beta_{1}(n)= & \sum_{\substack{u m p=n \\
u \leq \log ^{B} x \\
p \leq b}} h(u) \frac{g_{1}(m) g(p) \log p}{\log m p}, \\
\beta_{2}(n)= & \sum_{\substack{u r p=n \\
u \leq \log ^{B} x \\
r \leq b}} h(u) \frac{g_{1}(r) g(p) \log p}{\log r p}, \\
\beta(n)= & g(n)-\beta_{1}(n)-\beta_{2}(n) .
\end{aligned}
$$

The constants $A$ and $B$ will be chosen later; we pick $b$ to be $(\log x)^{6 A+15}$.

It follows from Lemma 5 of Elliott [4] that $\beta_{j}(n) \ll(\log x)^{(B+1) / 2}$, uniformly in $n, j$. Moreover, for $\varepsilon>0$, and $n>x^{\varepsilon}$,

$$
\begin{aligned}
& \beta_{1}(n) \ll \frac{1}{\log x} \sum_{\substack{u m p=n \\
u \leq \log ^{B} x \\
p \leq b}}|h(u)| \log p \ll \frac{1}{\log x} \sum_{\substack{u m p=n \\
u \leq \log ^{B} x \\
p \leq b}} h_{0}(u) \log p, \\
& \beta_{2}(n) \ll \frac{1}{\log x} \sum_{\substack{u r p=n \\
u \leq \log ^{B} x \\
r \leq b}} h_{0}(u) \log p .
\end{aligned}
$$

Here $h_{0}(u)$ is the multiplicative function defined by $h_{0}(p)=0, h_{0}\left(p^{2}\right)=3 / 2$, 
$h_{0}\left(p^{k}\right)=e$, if $k \geq 3$. Denote the above bounds for $\beta_{1}$ and $\beta_{2}$ by $\Theta_{1}$ and $\Theta_{2}$, respectively.

To realize the inequalities in (20), one examines the Euler product of $h * g_{1}$. It may be shown that for all positive integers $k$,

$$
h\left(p^{k}\right)=\sum_{u+v=k} \frac{(-g(p))^{u}}{u !} g\left(p^{v}\right) .
$$

The inequalities in (20) follow from this identity.

Let $z \leq x^{1 / 10}$. Then (19) leads to

$$
\begin{aligned}
\text { (21) } Q_{h} \ll & \frac{1}{\Psi_{\gamma}(x)} \int_{-1}^{1}(1-|t|) e^{-i t h} \sum_{\substack{d_{j} \leq z \\
d_{j} \mid P_{w, z}}} \lambda_{d_{1}} \lambda_{d_{2}} \sum_{\begin{array}{c}
n \leq x \\
\left(n, P_{y}, w\right)=1 \\
n(n+\gamma) \equiv 0\left(\left[d_{1}, d_{2}\right]\right)
\end{array}} \beta(n+a) d t \\
& +\sum_{m=1}^{2} \frac{1}{\Psi_{\gamma}(x)} \int_{-1}^{1}(1-|t|) e^{-i t h} \sum_{\substack{d_{j} \leq z \\
d_{j} \mid P_{w, z}}} \lambda_{d_{1}} \lambda_{d_{2}} \\
& \times \sum_{\substack{n \leq x \\
\left(n, P_{y, w}\right)=1 \\
n(n+\gamma) \equiv 0\left(\left[d_{1}, d_{2}\right]\right)}} \beta_{m}(n+a) d t+O\left(\frac{x^{1 / 10}}{\Psi_{\gamma}(x)}\right) .
\end{aligned}
$$

Denote the three sums above by $I_{0}, I_{1}, I_{2}$, respectively. We will show that $I_{0}$ is the only term making significant contribution. Define:

$$
\begin{aligned}
E(t)= & \sum_{\substack{d_{j} \leq z \\
d_{j} \mid P_{w, z}}} \lambda_{d_{1}} \lambda_{d_{2}} \sum_{\substack{n \leq x \\
\left(n(n+\gamma), P_{y}, w\right)=1 \\
n(n+\gamma) \equiv 0\left(\left[d_{1}, d_{2}\right]\right)}} \beta(n+a) \\
& -\sum_{\substack{d_{j} \leq z \\
d_{j} \mid P_{w, z}}} \lambda_{d_{1}} \lambda_{d_{2}} \frac{\varrho_{\gamma}\left(\left[d_{1}, d_{2}\right]\right)}{\phi\left(\left[d_{1}, d_{2}\right]\right)} \sum_{\substack{n \leq x \\
\left(n(n+\gamma), P_{y}, w\right)=1 \\
\left(n+a,\left[d_{1}, d_{2}\right]\right)=1}} \beta(n+a) .
\end{aligned}
$$

Then

$$
\begin{aligned}
I_{0}=\int_{-1}^{1} \frac{(1-|t|) e^{-i t h}}{\Psi_{\gamma}(x)} \sum_{\substack{d_{j} \leq z \\
d_{j} \mid P_{w, z}}} \lambda_{d_{1}} \lambda_{d_{2}} \frac{\varrho_{\gamma}\left(\left[d_{1}, d_{2}\right]\right)}{\phi\left(\left[d_{1}, d_{2}\right]\right)} \\
\times \sum_{\substack{n \leq x \\
\left(n(n+\gamma), P_{y, w}\right)=1 \\
\left(n+a,\left[d_{1}, d_{2}\right]\right)=1}} \beta(n+a) d t+\int_{-1}^{1} \frac{(1-|t|) e^{-i t h}}{\Psi_{\gamma}(x)} E(t) d t .
\end{aligned}
$$

Let $D$ be a typical value of $\left[d_{1}, d_{2}\right]$ with $d_{j} \leq z, d_{j} \mid P_{w, z}, j=1,2$. For each 
fixed $D$, the number of distinct choices of $d_{1}$ and $d_{2}$ such that $\left[d_{1}, d_{2}\right]=D$ is $3^{\omega(D)}$.

Since $\left|\lambda_{d_{j}}\right| \leq 1$ and $\beta(n) \ll(\log x)^{B+1 / 2}$ uniformly in $n$, it follows that

$$
\begin{aligned}
E(t) \ll & \sum_{\substack{\left.D \leq z^{2} \\
p|D \Rightarrow p>w \\
D, a| a-\gamma \mid\right)=1}} 3^{\omega(D)} \\
& \times\left|\sum_{\substack{n \leq x \\
\left(P_{\gamma}(n), P_{y, w}\right)=1 \\
P_{\gamma}(n) \equiv 0(D)}} \beta(n)-\frac{\varrho_{\gamma}(D)}{\phi(D)} \sum_{\substack{n \leq x \\
\left(P_{\gamma}(n), P_{y, w}\right)=1 \\
(n, D)=1}} \beta(n)\right|+O(\sqrt{x}),
\end{aligned}
$$

for $z \leq x^{1 / 10}$.

An application of Hölder's inequality shows that the main term of $E(t)$ is not more than

$$
(\log x)^{81 / 2}\left(\sum_{\substack{\left.D \leq z^{2} \\ p|D \Rightarrow p>w \\ D, a| a-\gamma \mid\right)=1}}\left(\frac{\phi(D)}{\varrho_{\gamma}(D)}\right)^{1 / 3}|F(t, D, x)|^{4 / 3}\right)^{3 / 4},
$$

where

$$
F(t, D, x)=\sum_{\substack{n \leq x \\\left(P_{\gamma}(n), P_{y, w}\right)=1 \\ P_{\gamma}(n) \equiv 0(D)}} \beta(n)-\frac{\varrho_{\gamma}(D)}{\phi(D)} \sum_{\substack{n \leq x \\\left(P_{\gamma}(n), P_{y, w}\right)=1 \\(n, D)=1}} \beta(n) .
$$

In the second product of (24), the term

$$
\left(\frac{\phi(D)}{\varrho_{\gamma}(D)}\right)^{1 / 3}\left|\sum_{\substack{n \leq x \\\left(P_{\gamma}(n), P_{y, w}\right)=1 \\ P_{\gamma}(n) \equiv 0(D)}} \beta(n)-\frac{\varrho_{\gamma}(D)}{\phi(D)} \sum_{\substack{n \leq x \\\left(P_{\gamma}(n), P_{y, w}\right)=1 \\(n, D)=1}} \beta(n)\right|^{1 / 3}
$$

is $\ll x^{1 / 3}(\log x)^{(B+1) / 6}$. Thus the second product in (24) may be bounded by the product of $x^{1 / 4}(\log x)^{(B+1) / 8}$ and the double sum

$$
\left(\sum_{\substack{\left.D \leq z^{2} \\ p|D \Rightarrow p>w \\ D, a| a-\gamma \mid\right)=1}}\left|\sum_{\substack{n \leq x \\\left(P_{\gamma}(n), P_{y, w}\right)=1 \\ P_{\gamma}(n) \equiv 0(D)}} \beta(n)-\frac{\varrho_{\gamma}(D)}{\phi(D)} \sum_{\substack{n \leq x \\\left(P_{\gamma}(n), P_{y, w}\right)=1 \\(n, D)=1}} \beta(n)\right|\right)^{3 / 4} .
$$

To estimate the last double sum, we shall employ the next two lemmas.

Lemma 8. Let $B \geq 0, A \geq 0, b=(\log x)^{6 A+15}$, and $0<\delta<1 / 2$. For any multiplicative function $g$ satisfying $|g(n)| \leq 1$ for all $n$, define the function 
$\beta$ as before. Then

$$
\begin{aligned}
\sum_{D_{1} D_{2} \leq x^{\delta}} \max _{\left(r, D_{1} D_{2}\right)=1}\left|\sum_{\substack{n \leq x \\
n \equiv r\left(D_{1} D_{2}\right)}} \beta(n)-\frac{1}{\phi\left(D_{2}\right)} \sum_{\substack{n \leq x \\
\left(n, D_{2}\right)=1 \\
n \equiv r\left(D_{1}\right)}} \beta(n)\right| \\
\ll x(\log x)^{-A}(\log \log x)^{2}+x w^{-1}(\log \log x)^{2} \\
\\
+x w^{-1 / 2}(\log x)^{5 / 2}(\log \log x)+x(\log x)^{5 / 2-B / 2},
\end{aligned}
$$

where $D_{1}$ is confined to those integers whose prime factors do not exceed $w$, and $D_{2}$ to integers whose prime factors exceed $w$. The implied constant depends at most upon $A$ and $B$.

Proof. This is Lemma 6 of [4].

Lemma 9. Let $B, A, b, g, \delta$ and $\beta$ be the same as in Lemma 8. Then

$$
\begin{array}{r}
\sum_{D_{1} D_{2} \leq x^{\delta}}\left|\sum_{\substack{n \leq x \\
P_{\gamma}(n) \equiv 0\left(D_{1} D_{2}\right)}} \beta(n)-\frac{\varrho_{\gamma}\left(D_{2}\right)}{\phi\left(D_{2}\right)} \sum_{\substack{n \leq x \\
\left(n, D_{2}\right)=1 \\
P_{\gamma}(n) \equiv 0\left(D_{1}\right)}} \beta(n)\right| \\
\ll x(\log x)^{(4-A) / 2} \log \log x+\frac{x}{\sqrt{w}}(\log x)^{A+6} \log \log x \\
+\frac{x}{\sqrt[4]{w}}(\log x)^{13 / 4}(\log \log x)^{1 / 2}+x(\log x)^{(13-B) / 4} .
\end{array}
$$

Here $D_{1}$ divides $P_{1, w}$, and $D_{2}$ is a squarefree integer such that if $p \mid D_{2}$, then $p>x$ and $p \nmid a(a-\gamma)$.

Proof. Let $b_{j}$ denote the solutions to $P_{\gamma}(n) \equiv 0\left(\bmod D_{1} D_{2}\right)$, where $1 \leq j \leq \varrho_{\gamma}\left(D_{1} D_{2}\right)$. Also, for $i=1$, 2 , let $c_{i k}, 1 \leq k \leq \varrho_{\gamma}\left(D_{i}\right)$, be the solutions to $P_{\gamma}(n) \equiv 0\left(\bmod D_{i}\right)$. To prove Lemma 9 , we essentially apply Lemma $8 \varrho_{\gamma}\left(D_{1} D_{2}\right)$ times. Therefore we shall only provide a sketch of the proof.

According to the Chinese Remainder Theorem, for each $j$ in the interval $\left[1, \varrho_{\gamma}\left(D_{1} D_{2}\right)\right]$, there exist $k \in\left[1, \varrho_{\gamma}\left(D_{1}\right)\right]$ and $l \in\left[1, \varrho_{\gamma}\left(D_{2}\right)\right]$ such that

$$
b_{j}=\alpha c_{1 k}+\beta c_{2 l},
$$

where $\alpha$ and $\beta$ are integers with

$$
\alpha \equiv\left\{\begin{array} { l l } 
{ 1 } & { ( \operatorname { m o d } D _ { 1 } ) , } \\
{ 0 } & { ( \operatorname { m o d } D _ { 2 } ) , }
\end{array} \text { and } \quad \beta \equiv \left\{\begin{array}{l}
0\left(\bmod D_{1}\right), \\
1\left(\bmod D_{2}\right) .
\end{array}\right.\right.
$$

It follows that 


$$
\begin{aligned}
\frac{\varrho_{\gamma}\left(D_{2}\right)}{\phi\left(D_{2}\right)} \sum_{\substack{n \leq x \\
\left(n, D_{2}\right)=1 \\
P_{\gamma}(n) \equiv 0\left(D_{1}\right)}} \beta(n) & =\frac{1}{\phi\left(D_{2}\right)} \sum_{k=1}^{\varrho_{\gamma}\left(D_{1}\right)} \sum_{i=1}^{\varrho_{\gamma}\left(D_{2}\right)} \sum_{\substack{n \leq x \\
\left(n, D_{2}\right)=1 \\
n \equiv c_{1}\left(D_{1}\right)}} \beta(n) \\
& =\frac{1}{\phi\left(D_{2}\right)} \sum_{j=1}^{\varrho_{\gamma}\left(D_{1} D_{2}\right)} \sum_{\substack{\left.n \leq x \\
n, D_{2}\right)=1 \\
n \equiv b_{j}\left(D_{1}\right)}} \beta(n) .
\end{aligned}
$$

The restrictions on $D_{1}$ and $D_{2}$ force $\left(b_{j}, D_{1} D_{2}\right)=1$ for each $j$. Otherwise, there is some prime dividing $\left(a, D_{1} D_{2}\right)$ or $\left(|a-\gamma|, D_{1} D_{2}\right)$, both of which are impossible. Therefore the sum to be estimated in Lemma 9 may be bounded by

$$
\sum_{D_{1} D_{2} \leq x^{\delta}} \varrho_{\gamma}\left(D_{1} D_{2}\right) \max _{\left(b, D_{1} D_{2}\right)=1}\left|\sum_{\substack{n \leq x \\ n \equiv b\left(D_{1} D_{2}\right)}} \beta(n)-\frac{1}{\phi\left(D_{2}\right)} \sum_{\substack{n \leq x \\\left(n, D_{2}\right)=1 \\ n \equiv b\left(D_{1}\right)}} \beta(n)\right| .
$$

The Cauchy-Schwarz inequality shows that the previous term is not greater than the product of $O\left(\log ^{4} x\right)$ and the square root of

$$
\sum_{D_{1} D_{2} \leq x^{\delta}} \phi\left(D_{1} D_{2}\right) \max _{\left(b, D_{1} D_{2}\right)=1}\left|\sum_{\substack{n \leq x \\ n \equiv b\left(D_{1} D_{2}\right)}} \beta(n)-\frac{1}{\phi\left(D_{2}\right)} \sum_{\substack{n \leq x \\\left(n, D_{2}=1 \\ n \equiv b\left(D_{1}\right)\right.}} \beta(n)\right|^{2} .
$$

Since

$$
\phi\left(D_{1} D_{2}\right)\left|\sum_{\substack{n \leq x \\ n \equiv b\left(D_{1} D_{2}\right)}} \beta(n)-\frac{1}{\phi\left(D_{2}\right)} \sum_{\substack{n \leq x \\\left(n, D_{2}\right)=1 \\ n \equiv b\left(D_{1}\right)}} \beta(n)\right| \ll x
$$

the product is

$$
\ll\left(x \log ^{4} x\right) \sum_{D_{1} D_{2} \leq x^{\delta}} \max _{\left(b, D_{1} D_{2}\right)=1}\left|\sum_{\substack{n \leq x \\ n \equiv b\left(D_{1} D_{2}\right)}} \beta(n)-\frac{1}{\phi\left(D_{2}\right)} \sum_{\substack{n \leq x \\\left(n, D_{2}=1 \\ n \equiv b\left(D_{1}\right)\right.}} \beta(n)\right| .
$$

Take square root and utilize Lemma 8 to estimate the remaining double sum. Lemma 9 now follows easily.

Proof of Theorem 1 (continued). Select $B=2 A+5$ and $w>(\log x)^{2 A+14}$. Then Lemma 9 asserts that the second product of $(24)$ is

$$
\ll x^{1 / 4}(\log x)^{(B+1) / 8}\left(x(\log x)^{3-A / 2}\right)^{3 / 4} \ll x(\log x)^{3-A / 8} .
$$


Therefore, inequalities (23) and (24) give the bounds

$$
\begin{aligned}
& |E(t)| \ll x(\log x)^{87 / 2-A / 8}+\sqrt{x} \\
& \frac{1}{\Psi_{\gamma}(x)} \int_{-1}^{1}(1-|t|) e^{-i t h} E(t) d t \ll \frac{x}{\Psi_{\gamma}(x)}(\log x)^{87 / 2-A / 8} .
\end{aligned}
$$

Having successfully dealt with the congruence condition in the innermost sum of $I_{0}$, we now switch back from $\beta$ to $g$. We used $\beta$ because Lemmas 8 and 9 are stated in terms of $\beta$, not $g$. From (22) and (25),

$$
\begin{aligned}
I_{0}= & \int_{-1}^{1} \frac{(1-|t|) e^{-i t h}}{\Psi_{\gamma}(x)} \\
& \times \sum_{\substack{d_{j} \leq z \\
d_{j} \mid P_{w, z}}} \lambda_{d_{1}} \lambda_{d_{2}} \frac{\varrho_{\gamma}\left(\left[d_{1}, d_{2}\right]\right)}{\phi\left(\left[d_{1}, d_{2}\right]\right)} \sum_{\begin{array}{c}
n \leq x \\
\left(n(n+\gamma), P_{y, w}\right)=1 \\
\left(n+a,\left[d_{1}, d_{2}\right]\right)=1
\end{array}} g(n+a) d t \\
& -K_{1}-K_{2}+O\left(\frac{x}{\Psi_{\gamma}(x)}(\log x)^{87 / 2-A / 8}\right),
\end{aligned}
$$

where for $m=1,2, K_{m}$ is defined to be

$$
\int_{-1}^{1} \frac{(1-|t|) e^{-i t h}}{\Psi_{\gamma}(x)} \sum_{\substack{d_{j} \leq z \\
d_{j} \mid P_{w, z}}} \lambda_{d_{1}} \lambda_{d_{2}} \frac{\varrho_{\gamma}\left(\left[d_{1}, d_{2}\right]\right)}{\phi\left(\left[d_{1}, d_{2}\right]\right)} \sum_{\begin{array}{c}
n \leq x \\
\left(n(n+\gamma), P_{y, w}=1\right. \\
\left(n+a,\left[d_{1}, d_{2}\right]\right)=1
\end{array}} \beta_{m}(n+a) d t .
$$

We next show that the two $K_{m}$ terms are also small. Hence, the main contribution to an upper bound for $Q_{h}$ will come from the first term in (26).

It follows easily from the definition that

$$
K_{1} \ll \max _{|t| \leq 1} \sum_{\substack{n \leq x \\\left(n(n+\gamma), P_{y, w}\right)=1}} \frac{\left|\beta_{1}(n+a)\right|}{\Psi_{\gamma}(x)}\left|\sum_{\substack{d_{j} \leq z \\ d_{j} \mid P_{w, z} \\\left(d_{j}, n+a\right)=1}} \lambda_{d_{1}} \lambda_{d_{2}} \frac{\varrho_{\gamma}\left(\left[d_{1}, d_{2}\right]\right)}{\phi\left(\left[d_{1}, d_{2}\right]\right)}\right| .
$$

In the innermost sum above, we wish to replace $\phi\left(\left[d_{1}, d_{2}\right]\right)$ with $\left[d_{1}, d_{2}\right]$. Also, we would like to sever the relationship between the $d_{j}$ 's and $n$. Once both of these tasks are accomplished, the $\lambda$ 's may be chosen independent of $n$ to minimize the quadratic form. We will then be able to utilize the sieve results stated in Lemma 2.

Since

$$
\frac{1}{\phi(D)}-\frac{1}{D} \leq \frac{1}{\phi(D)} \sum_{\substack{p \mid D \\ p>w}} \frac{1}{p} \quad \text { and } \quad \sum_{u} \frac{|h(u)|}{u} \ll 1
$$


the error introduced in replacing $\phi\left(\left[d_{1}, d_{2}\right]\right)$ with $\left[d_{1}, d_{2}\right]$ is

$$
\ll \frac{x}{w \Psi_{\gamma}(x)}\left(\frac{\log x}{\log \log x}\right)^{6} .
$$

The error term arising from the removal of the condition $\left(d_{j}, n+a\right)=1$ is

$$
\ll \frac{1}{w} \log ^{7} x \text {. }
$$

Having severed the connection between the $d_{j}$ 's and $n$, we may apply Lemma 2 to bound the sum over the $d_{j}$ 's. Together, inequalities (20) and (27) show that

$$
\begin{aligned}
K_{1} \ll & \frac{1}{\Psi_{\gamma}(x)} \sum_{x^{\varepsilon}<n \leq x} \Theta_{1}(n+a)\left(\Pi_{1}\left(\frac{\log \log x}{\log x}\right)^{2}+\frac{\log ^{7} x}{w}\right) \\
& +\frac{1}{\Psi_{\gamma}(x)} \sum_{\substack{n \leq x^{\varepsilon} \\
\left(n(n+\gamma), P_{y, w}\right)=1}}(\log x)^{(B+1) / 2} \\
& \times\left(\Pi_{1}\left(\frac{\log \log x}{\log x}\right)^{2}+\frac{\log ^{7} x}{w}\right) \\
& +\frac{x}{w \Psi_{\gamma}(x)}\left(\frac{\log x}{\log \log x}\right)^{6},
\end{aligned}
$$

where $\Pi_{1}$ is defined in Lemma 2 . Recall that $\Pi_{1} \ll \log \log x$ if $\gamma<x$ and $w>\log x$.

Choose $w$ to be greater than $\log ^{9} x$. Then one easily verifies that the second sum in $(28)$ is

$$
\ll \frac{x^{\varepsilon}}{\Psi_{\gamma}(x)}(\log \log x)^{3}(\log x)^{(B-3) / 2} .
$$

As for the first sum in (28), it is

$$
\begin{aligned}
& \ll \frac{1}{\Psi_{\gamma}(x)} \cdot \frac{(\log \log x)^{3}}{\log ^{3} x} \sum_{\substack{u \leq \log ^{B} x \\
p \leq\left(\log x^{6}\right)^{6+15}}}\left|h_{0}(u)\right| \log p \sum_{m \leq(x-a) /(u p)} 1 \\
& \ll \frac{x}{\Psi_{\gamma}(x)} \cdot \frac{(\log \log x)^{4}}{\log ^{3} x} .
\end{aligned}
$$

Together, the bounds for the first and the second term of (28) yield

$$
\begin{aligned}
K_{1} & \ll \frac{x(\log \log x)^{4}}{\Psi_{\gamma}(x) \log ^{3} x}+\frac{x^{\varepsilon}(\log \log x)^{3}}{\Psi_{\gamma}(x)(\log x)^{(3-B) / 2}}+\frac{x}{w \Psi_{\gamma}(x)}\left(\frac{\log x}{\log \log x}\right)^{6} \\
& \ll \frac{x(\log \log x)^{4}}{\Psi_{\gamma}(x) \log ^{3} x} .
\end{aligned}
$$


The $K_{2}$ term may be bounded similarly. The only difference is that we must use $\Theta_{2}$, as defined in (20), as an upper bound for $\beta_{2}$. Since $\beta_{2}(n) \ll$ $\Theta_{2}(n)$ for $n>x^{\varepsilon}, K_{2}$ satisfies an estimate identical to (28) with $\Theta_{1}$ replaced by $\Theta_{2}$. The term analogous to the sum involving $\Theta_{1}$ is

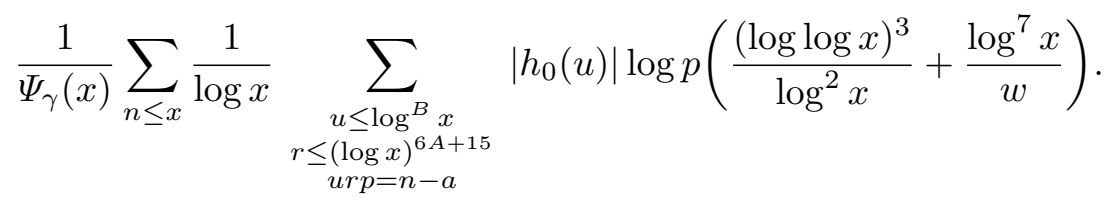

This double sum, in turn, is

$$
\begin{aligned}
& \ll \frac{1}{\Psi_{\gamma}(x)} \cdot \frac{(\log \log x)^{3}}{\log ^{3} x} \sum_{\substack{u \leq \log ^{B} x \\
r \leq(\log x)^{6 A+15}}} h_{0}(u) \sum_{p \leq(x-a) /(u r)} \log p \\
& \ll \frac{x}{\Psi_{\gamma}(x)} \cdot \frac{(\log \log x)^{4}}{\log ^{3} x} .
\end{aligned}
$$

Hence

$$
\begin{aligned}
K_{2} & \ll \frac{x(\log \log x)^{4}}{\Psi_{\gamma}(x) \log ^{3} x}+\frac{x^{\varepsilon}(\log \log x)^{3}}{\Psi_{\gamma}(x)(\log x)^{(3-B) / 2}}+\frac{x}{w \Psi_{\gamma}(x)}\left(\frac{\log x}{\log \log x}\right)^{6} \\
& \ll \frac{x}{\Psi_{\gamma}(x)} \cdot \frac{(\log \log x)^{4}}{\log ^{3} x} .
\end{aligned}
$$

This completes the study of $I_{0}$ for the moment. We now turn our attention to $I_{1}$ and $I_{2}$. The $I_{1}$ term may be estimated in manner similar to that used for the $K_{1}$ term. In fact, we have

$$
I_{1} \ll \frac{1}{\Psi_{\gamma}(x)} \cdot \frac{(\log \log x)^{4}}{\log ^{3} x} .
$$

Bounding the $I_{2}$ term requires a combination of the techniques employed in estimating the $E(t)$ term and the $K_{2}$ term. From its definition stated in (21), $I_{2}$ may be estimated by

$$
\begin{aligned}
& \frac{1}{\Psi_{\gamma}(x)} \sum_{x^{\varepsilon}<n \leq x} \Theta_{2}(n+a)\left(\sum_{\substack{d \leq z \\
d \mid\left(P_{w, z}, n(n+\gamma)\right)}} \lambda_{d}\right)^{2} \\
& \quad+\frac{1}{\Psi_{\gamma}(x)} \sum_{\substack{n \leq x^{\varepsilon} \\
\left(n(n+\gamma), P_{y, w}\right)=1}}(\log x)^{(B+1) / 2}\left(\sum_{\substack{d \leq z \\
d \mid\left(P_{w, z}, n(n+\gamma)\right)}} \lambda_{d}\right)^{2} .
\end{aligned}
$$

We have encountered the second term of (32) earlier in bounding the $K_{1}$ term. It is dominated by $O\left(\sqrt{x} / \Psi_{\gamma}(x)\right)$.

Recall the definition of $\Theta_{2}(n)$. The first double sum in (32) has the 
representation

(33)

$$
\begin{aligned}
\frac{1}{\Psi_{\gamma}(x) \log x} \sum_{\substack{u \leq \log ^{B} x \\
r \leq(\log x)^{6 A+15}}} h_{0}(u) \sum_{\substack{d_{j} \leq z \\
d_{j} \mid P_{w, z}}} \lambda_{d_{1}} \lambda_{d_{2}} \frac{x \varrho_{\gamma}\left(\left[d_{1}, d_{2}\right]\right)}{u r \phi\left(\left[d_{1}, d_{2}\right]\right)} \\
+\frac{1}{\Psi_{\gamma}(x) \log x} \sum_{\substack{u \leq \log ^{B} x \\
r \leq(\log x)^{6 A+15}}} h_{0}(u) F(x, t),
\end{aligned}
$$

where

$$
F(x, t)=\sum_{\substack{d_{j} \leq z \\ d_{j} \mid P_{w, z}}} \lambda_{d_{1}} \lambda_{d_{2}}\left(\sum_{\substack{p \leq x /(u r) \\ P_{\gamma}(p u r) \equiv 0\left(\left[d_{1}, d_{2}\right]\right)}} \log p-\frac{x \varrho_{\gamma}\left(\left[d_{1}, d_{2}\right]\right)}{u r \phi\left(\left[d_{1}, d_{2}\right]\right)}\right) .
$$

In bounding the first term of (33), we proceed as with the $K_{1}$ and $K_{2}$ terms. First, replacing $\phi\left(\left[d_{1}, d_{2}\right]\right)$ with $\left[d_{1}, d_{2}\right]$ creates an error which is

$$
\ll \frac{x}{w \Psi_{\gamma}(x)}\left(\frac{\log x}{\log \log x}\right)^{6} .
$$

After the replacement, Lemma 2 yields an upper bound of

$$
\ll \frac{x}{\Psi_{\gamma}(x)} \cdot \frac{(\log \log x)^{4}}{\log ^{3} x}
$$

for the first double sum in (33).

The function $F(x, t)$ may be bounded in a fashion similar to that applied to the $E(t)$ term. Let $D$ denote a typical value of $\left[d_{1}, d_{2}\right]$. Then, by selecting $w$ such that $w>(\log x)^{6 A+15}>\log ^{B} x$, we have $(D, u r)=1$ for each $D$. It follows that

$$
|F(x, t)| \leq \sum_{\substack{\left.D \leq z^{2} \\ q|D \Rightarrow q>w \\ D, a| a-\gamma \mid\right)=1}} 3^{\omega(D)} \varrho_{\gamma}(D) \max _{b}\left|\sum_{\substack{p \leq x /(u r) \\ p \equiv b(D)}} \log p-\frac{x \varrho_{\gamma}(D)}{u r \phi(D)}\right|,
$$

where $b$ runs through the $\varrho_{\gamma}(D)$ solutions to $P_{\gamma}($ nur $) \equiv 0(\bmod D)$ with $(n, D)=1$.

The Cauchy-Schwarz inequality shows that

$$
\begin{aligned}
|F(x, t)|^{2} \ll & \left(\frac{\log x}{\log \log x}\right)^{36} \\
& \times\left(\sum_{\substack{\left.D \leq z^{2} \\
q|D \Rightarrow q>w \\
D, a| a-\gamma \mid\right)=1}} \phi(D) \max _{b}\left|\sum_{\substack{p \leq x /(u r) \\
p \equiv b(D)}} \log p-\frac{x}{u r \phi(D)}\right|^{2}\right) .
\end{aligned}
$$


For each $D$ and $b$ with $0 \leq b<D$,

$$
\phi(D)\left|\sum_{\substack{p \leq x / u r) \\ p \equiv b(D)}} \log p-\frac{x}{u r \phi(D)}\right| \leq \frac{x \log x}{u r}+D \log x+\frac{x}{u r} .
$$

Since $D \leq x^{1 / 3}$, the last expression is dominated by $(x \log x) /(u r)$.

It now follows from Bombieri and Vinogradov's celebrated result, Theorem 17 of [1], that

$$
\begin{aligned}
|F(x, t)|^{2} \ll & \left(\frac{\log x}{\log \log x}\right)^{36}\left(\frac{x \log x}{u r}\right) \\
& \times\left(\sum_{\substack{\left.D \leq z^{2} \\
q|D \Rightarrow q>w \\
D, a| a-\gamma \mid\right)=1}} \max _{b}\left|\sum_{\substack{p \leq x /(u r) \\
p \equiv b(D)}} \log p-\frac{x}{u r \phi(D)}\right|\right) \\
\ll & \left(\frac{x}{u r}\right)^{2} \frac{(\log x)^{37-c}}{(\log \log x)^{36}} .
\end{aligned}
$$

Thus the second sum of (33) is

$$
\ll \frac{x(\log x)^{(35-c) / 2}}{\Psi_{\gamma}(x)(\log \log x)^{17}} .
$$

According to Bombieri and Vinogradov's Theorem, $c$ may be chosen arbitrarily large. Also, if $w>\log ^{9} x$, inequalities (32) through (35) yield the following bound:

$$
I_{2} \ll \frac{x}{\Psi_{\gamma}(x)} \cdot \frac{(\log \log x)^{4}}{\log ^{3} x} .
$$

Having obtain estimates for the three sums, $I_{0}, I_{1}, I_{2}$, we now return to the study of $Q_{h}$. According to inequalities (21), (22), (25) and (26),

$$
\begin{aligned}
Q_{h} \ll & \int_{-1}^{1} \frac{(1-|t|) e^{-i t h}}{\Psi_{\gamma}(x)} \\
& \times \sum_{\substack{d_{j} \leq z \\
d_{j} \mid P_{w, z}}} \lambda_{d_{1}} \lambda_{d_{2}} \frac{\varrho_{\gamma}\left(\left[d_{1}, d_{2}\right]\right)}{\phi\left(\left[d_{1}, d_{2}\right]\right)} \sum_{\substack{n \leq x \\
\left(n(n+\gamma), P_{y, w}\right)=1 \\
\left(n+a,\left[d_{1}, d_{2}\right]\right)=1}} g(n+a) d t \\
& +K_{1}+K_{2}+\frac{x}{\Psi_{\gamma}(x)}(\log x)^{87 / 2-A / 8}+I_{1}+I_{2} .
\end{aligned}
$$

Inequalities (29), (30), (31), and (35) give bounds for $K_{1}, K_{2}, I_{1}, I_{2}$, respec- 
tively. Therefore,

$$
\begin{aligned}
Q_{h} \ll & \int_{-1}^{1} \frac{(1-|t|) e^{-i t h}}{\Psi_{\gamma}(x)} \\
& \times \sum_{\substack{d_{j} \leq z \\
d_{j} \mid P_{w, z}}} \lambda_{d_{1}} \lambda_{d_{2}} \frac{\varrho_{\gamma}\left(\left[d_{1}, d_{2}\right]\right)}{\phi\left(\left[d_{1}, d_{2}\right]\right)} \sum_{\substack{n \leq x \\
\left(n(n+\gamma), P_{y, w}\right)=1 \\
\left(n+a,\left[d_{1}, d_{2}\right]\right)=1}} g(n+a) d t \\
& +O\left(\frac{x(\log \log x)^{4}}{\Psi_{\gamma}(x) \log ^{3} x}\right),
\end{aligned}
$$

provided $A$ is chosen to be a constant greater than 372 .

In the main term of $(37)$, we once again replace $\phi\left(\left[d_{1}, d_{2}\right]\right)$ with $\left[d_{1}, d_{2}\right]$ and remove the condition of relative primality $\left(\left[d_{1}, d_{2}\right], n+a\right)=1$. Two error terms are introduced:

$$
\begin{gathered}
\int_{-1}^{1} \frac{(1-|t|) e^{-i t h}}{\Psi_{\gamma}(x)} \sum_{\substack{d_{j} \leq z \\
d_{j} \mid P_{w}, z}} \lambda_{d_{1}} \lambda_{d_{2}}\left(\frac{\varrho_{\gamma}\left(\left[d_{1}, d_{2}\right]\right)}{\phi\left(\left[d_{1}, d_{2}\right]\right)}-\frac{\varrho_{\gamma}\left(\left[d_{1}, d_{2}\right]\right)}{\left[d_{1}, d_{2}\right]}\right) \\
\times \sum_{\begin{array}{c}
n \leq x \\
\left(n+a,\left[d_{1}, d_{2}\right]\right)=1 \\
\left(n(n+\gamma), P_{y, w}\right)=1
\end{array}} g(n+a) d t,
\end{gathered}
$$

and

$$
\int_{-1}^{1} \frac{(1-|t|) e^{-i t h}}{\Psi_{\gamma}(x)} \sum_{\substack{d_{j} \leq z \\ d_{j} \mid P_{w, z}}} \lambda_{d_{1}} \lambda_{d_{2}} \frac{\varrho_{\gamma}\left(\left[d_{1}, d_{2}\right]\right)}{\left[d_{1}, d_{2}\right]} \sum_{\substack{n \leq x \\\left(n+a,\left[d_{1}, d_{2}\right]\right)>1 \\\left(n(n+\gamma), P_{y}, w\right)=1}} g(n+a) d t .
$$

Denote these two error terms by $E_{1}$ and $E_{2}$, respectively.

A direct argument shows that

$$
E_{1} \ll \frac{x}{w \Psi_{\gamma}(x)} \log ^{6} x .
$$

As for the second error,

$$
E_{2} \ll \frac{1}{\Psi_{\gamma}(x)} \sum_{q \mid P_{w, z^{2}}} \sum_{\substack{D \leq z^{2} \\ p \mid D \Rightarrow p>w \\ D \equiv 0(q)}} \frac{6^{\omega(D)}}{D} \sum_{\substack{n \leq x \\ n \equiv 0(q)}} 1
$$

where $q$ is prime. Express $D$ as $q m$. Then the Cauchy-Schwarz inequality 
shows

$$
E_{2} \ll\left(\frac{x}{\Psi_{\gamma}(x)}+\frac{z^{2}}{\Psi_{\gamma}(x)}\right) \sum_{q \mid P_{w, z^{2}}}\left(\frac{1}{q}\left(\log ^{36} x\right)^{1 / 2}\left(\sum_{m \leq z^{2} / q} \frac{1}{m q}\right)^{1 / 2}\right) .
$$

The last quantity is

$$
\ll \frac{x \log ^{20} x}{\sqrt{w} \Psi_{\gamma}(x)} .
$$

Both terms, $E_{1}$ and $E_{2}$, are smaller than the error term in the bound for $Q_{h}$ stated in (37), provided $w>\log ^{40} x$. Furthermore, because the integral in the main term of (37) is nonnegative, the sum over $d_{j}$ 's may be bounded using Lemma 2. Consequently, it follows from (37) that

$$
\begin{aligned}
Q_{h} \ll & \frac{\Pi_{1}}{\Psi_{\gamma}(x)}\left(\frac{\log \log x}{\log x}\right)^{2} \\
& \times \int_{-1}^{1}(1-|t|) e^{-i t h} \sum_{\substack{n \leq x \\
\left(n(n+\gamma), P_{y, w}\right)=1}} g(n+a) d t \\
& +O\left(\frac{x(\log \log x)^{4}}{\Psi_{\gamma}(x) \log ^{3} x}\right) .
\end{aligned}
$$

Apply the results of Lemma 7 to the inequality (38). There are two cases to consider, according to whether there are exceptional moduli or not. Let us consider the former situation, which is the more difficult of the two. In view of the second estimate of Lemma 7 ,

$$
\begin{aligned}
Q_{h} \ll & \frac{\Pi_{1}}{\Psi_{\gamma}(x)}\left(\frac{\log \log x}{\log x}\right)^{2} \\
& \times\left|\int_{-1}^{1}(1-|t|) e^{-i t h} H\left(P_{y, w}\right) \sum_{n \leq x} g(n) G\left(P_{y, w}, n\right) d t\right| \\
& +\frac{\Pi_{1}}{\Psi_{\gamma}(x)}\left(\frac{\log \log x}{\log x}\right)^{2} \mid \int_{-1}^{1}(1-|t|) e^{-i t h} H\left(P_{y, w}\right) \frac{\mu\left(D_{0}\right)}{D_{0}} \\
& \times \prod_{p \mid D_{0}}\left(\frac{p}{p-2}\right) \prod_{p \mid D_{0}}\left(\frac{p}{p-3}\right) \sum_{p \nmid \gamma}^{\varrho_{\gamma}\left(D_{0}\right)} \bar{\chi}\left(b_{j}\right) \\
& \times \sum_{n \leq x} \chi(n) g(n) G\left(\frac{P_{y, w}}{D_{0}}, n\right) d t \mid
\end{aligned}
$$




$$
\begin{aligned}
& +\frac{1}{\Psi_{\gamma}(x)} \cdot \frac{(\log \log x)^{3}}{\log ^{2} x} \\
& \times\left|\int_{-1}^{1}(1-|t|) e^{-i t h} \frac{x}{(\log x)^{1 / 20}} d t\right|+\frac{x(\log \log x)^{4}}{\Psi_{\gamma}(x) \log ^{3} x} .
\end{aligned}
$$

The quantities $D_{0}, \chi, \varrho_{\gamma}\left(D_{0}\right), b_{j}, G$, and $H$ are as defined in Lemma 7 .

Let

$$
U(x, \lambda)=\sum_{p \leq x} \frac{1}{p} \min \left(1,(f(p)-\lambda \log p)^{2}\right) .
$$

Ruzsa breaks his argument into two main cases. Suppose

$$
U(x, \lambda) \geq \frac{1}{100} \log \log x
$$

for all real $\lambda$ 's with $|\lambda| \leq \log ^{2} x$, in the first case. Although this was not explicitly stated, Ruzsa actually placed such a restriction on the $\lambda$ 's (see page 221 of [7]). Moreover, changing the constant from 1/10 to 1/100 will only increase the implied constant in the final upper bound. In view of Lemma 1,

$$
\begin{aligned}
\sum_{n \leq x} \chi(n) g(n) G( & \left.P_{0}, n\right) \\
& \ll x \exp \left(-\frac{1}{8} \min _{|u| \leq \log x} \sum_{p \leq x} \frac{1}{p}\left(1-\operatorname{Re}\left(\frac{g(p) \chi(p)}{p^{i u}}\right)\right)\right),
\end{aligned}
$$

where $\chi$ is any Dirichlet character. In particular, (40) holds when $\chi$ is the exceptional primitive character modulo $D_{0}$ defined in Lemma 7 and when $\chi$ is the principal character modulo one.

For reals $u$ and $t$ and a Dirichlet character $\psi(\bmod D)$, let

$$
m(u, \psi, t)=\sum_{\substack{p \leq x \\(p, D)=1}} \frac{1}{p}\left(1-\operatorname{Re}\left(\frac{g(p) \psi(p)}{p^{i u}}\right)\right)
$$

and

$$
M(t)=\min _{\substack{|u| \leq \log x \\ \psi}} m(u, \psi, t),
$$

where the minimum is taken over all $|u| \leq \log x$ and characters $\psi$ with moduli not exceeding $Q=\exp (\log \log x)^{3}$. Thus, whether $\chi$ is the principal character modulo one or $\chi$ is the exceptional character modulo $D_{0}$ with $D_{0} \leq Q$, we have

$$
-\frac{1}{8} \sum_{p \leq x} \frac{1}{p}\left(1-\operatorname{Re}\left(\frac{g(p) \chi(p)}{p^{i u}}\right)\right) \leq-\frac{1}{8} M(t) .
$$


Moreover, observe that for any $D_{0} \mid P_{y, w}$,

$$
\left|\frac{\varrho_{\gamma}\left(D_{0}\right)}{D_{0}}\right| \prod_{\substack{p \mid D_{0} \\ p \nmid \gamma}}\left(\frac{p}{p-3}\right) \prod_{\substack{p\left|D_{0} \\ p\right| \gamma}}\left(\frac{p}{p-2}\right)=\prod_{\substack{p \mid D_{0} \\ p \nmid \gamma}}\left(\frac{2}{p-3}\right) \prod_{\substack{p\left|D_{0} \\ p\right| \gamma}}\left(\frac{1}{p-2}\right) .
$$

The last products are not greater than one.

Lastly, recall the definitions of $H\left(P_{y, w}\right)$ stated just before Lemma 6 . A direct argument shows that

$$
H\left(P_{y, w}\right) \ll \frac{1}{(\log \log x)^{2}} \prod_{\substack{y<p \leq w \\ p \mid a(a-\gamma)}}\left(\frac{p-1}{p-3}\right) \prod_{\substack{p\left|P_{y, w} \\ p\right| \gamma}}\left(\frac{p-2}{p-3}\right) .
$$

Then inequality (40) in conjunction with the three remarks above allows us to rewrite inequality (39) as

$$
\begin{aligned}
Q_{h} \ll & \frac{x \Pi_{1}}{\Psi_{\gamma}(x)}\left(\frac{\log \log x}{\log x}\right)^{2} H\left(P_{y, w}\right) \int_{-1}^{1} \exp \left(\frac{-M(t)}{8}\right) d t \\
& +\frac{x(\log \log x)^{3}}{\Psi_{\gamma}(x)(\log x)^{41 / 20}}+\frac{x(\log \log x)^{4}}{\Psi_{\gamma}(x) \log ^{3} x} \\
\ll & \frac{x}{\Psi_{\gamma}(x)} \cdot \frac{\Pi_{1}}{\log ^{2} x} \prod_{\substack{y<p \leq w \\
p \mid a(a-\gamma)}}\left(\frac{p-1}{p-3}\right) \prod_{p \mid P_{y, w}}\left(\frac{p-2}{p-3}\right) \int_{-1}^{1} \exp \left(\frac{-M(t)}{8}\right) d t \\
& +\frac{x(\log \log x)^{3}}{\Psi_{\gamma}(x)(\log x)^{41 / 20}} .
\end{aligned}
$$

A similar argument shows that the above inequality continues to hold when there are no exceptional moduli.

Recall the definitions of $\Psi_{\gamma}(x)$ stated at the beginning of the paper. Then, whether or not there are exceptional moduli,

$$
Q_{h} \ll \Pi_{2} \int_{-1}^{1} \exp \left(-\frac{1}{8} M(t)\right) d t
$$

where $\Pi_{2}$ is the following product:

$$
\begin{array}{r}
\prod_{p>2}\left(1-\frac{1}{(p-1)^{2}}\right)^{-1} \prod_{\substack{p \mid \gamma \\
p>2}}\left(\frac{p-2}{p-1}\right) \prod_{\substack{p\left|P_{y, w} \\
p\right| \gamma}}\left(\frac{p-2}{p-3}\right) \prod_{\substack{p\left|P_{w, z} \\
p\right| \gamma}}\left(\frac{p-1}{p-2}\right) \\
\times \prod_{\substack{y<p \leq w \\
p \mid a(a-\gamma)}}\left(\frac{p-1}{p-3}\right) \prod_{\substack{w<p \leq z \\
p \mid a(a-\gamma)}}\left(\frac{p}{p-2}\right) .
\end{array}
$$


The last bound on $Q_{h}$ is slightly weaker than inequality (15) of Elliott [4]. The only difference lies in the definition of $M(t)$. In Elliott's paper, the quantity $M(t)$ is the minimum value of $m(u, \psi, t)$ taken over all characters $\psi$ with moduli not exceeding $\log x$ rather than $Q$ as in our case. Nevertheless, we may follow Elliott's argument, which leads to the conclusion

$$
Q_{h} \ll \Pi_{2}(1+W(x))^{-1 / 2}
$$

in our situation as well. For $w \geq \log x$ and $\gamma \leq x, \Pi_{2}$ may be replaced with the simpler expression stated in the theorem. Theorem 1 is thus established in the first case.

In the second case, suppose there exists $\left|\lambda_{0}\right| \leq \log ^{2} x$ such that $U\left(x, \lambda_{0}\right)$ $\leq \frac{1}{100} \log \log x$. For $|t| \leq 1$ and $g=e^{i t f}$,

$$
\left|1-\frac{g(p)}{p^{i \lambda t}}\right| \leq \min (2,|f(p)-\lambda \log p|) .
$$

It is not difficult to verify that

$$
\frac{1}{p} \min (2,|f(p)-\lambda \log p|)^{2} \leq \frac{4}{p} \min \left(1,|f(p)-\lambda \log p|^{2}\right) .
$$

Hence the Cauchy-Schwarz inequality gives

$$
\begin{aligned}
\sum_{p \leq x} \frac{1}{p}\left(1-\operatorname{Re}\left(\frac{g(p)}{p^{i \lambda_{0} t}}\right)\right) & \leq \sum_{p \leq x} \frac{1}{p} \min \left(2,\left|f(p)-\lambda_{0} \log p\right|\right) \\
& \leq\left(4 U\left(x, \lambda_{0}\right) \sum_{p \leq x} \frac{1}{p}\right)^{1 / 2} .
\end{aligned}
$$

Since $U\left(x, \lambda_{0}\right) \leq \frac{1}{100} \log \log x$, this last quantity is

$$
\leq\left(\frac{1}{5}+o(1)\right) \log \log x
$$

Because $Q=\exp \left((\log \log x)^{3}\right)$ and $1-\operatorname{Re}\left(g(p) p^{-i \lambda_{0} t}\right) \geq 0$,

$$
\sum_{Q<p \leq x} \frac{1}{p}\left(1-\operatorname{Re}\left(\frac{g(p)}{p^{i \lambda_{0} t}}\right)\right) \leq\left(\frac{1}{5}+o(1)\right) \log \log x<\frac{1}{4} \log \left(\frac{\log x}{\log Q}\right)-c,
$$

where $c$ is the constant appearing in Lemma 3 .

Therefore, if there exists $\lambda_{0}$ such that $U\left(x, \lambda_{0}\right) \leq \frac{1}{100} \log \log x$, then the characters for which inequality (2) in Lemma 3 holds are all principal. It follows that the first estimate in Lemma 7 is valid without exception.

Without any exceptional moduli, the analogous form of inequality (39) is

$$
Q_{h} \ll \frac{\Pi_{2}}{x} \int_{-1}^{1}(1-|t|) e^{-i t h} \sum_{n \leq x} e^{i t f(n)} G\left(P_{y, w}, n\right) d t+c \frac{(\log \log x)^{2}}{(\log x)^{1 / 20}},
$$

for some absolute constant $c$. 
The last integral is similar to the one studied by Ruzsa (see $\S 3$, pp. 218219 , of [7]). The extraneous product factor of $G\left(P_{y, w}, n\right)$ may be carried through Ruzsa's entire argument with no difficulty. As in Ruzsa's paper, we therefore reach

$$
Q_{h} \ll \prod_{p \mid a(\gamma-a)}\left(1+\frac{2}{p}\right) \frac{1}{\sqrt{W(x)}} .
$$

The proof of Theorem 1 is thus completed.

5. Proof of Theorem 2. Theorem 2 is now a simple consequence of Theorem 1 and inequality (1). Employ a convolution argument similar to the one used in Lemma 4. Define multiplicative functions $v$ and $u$ by

$$
\sum_{d \mid \gamma} v(d)=\prod_{\substack{p\left|P_{y, w} \\ p\right| \gamma}}\left(\frac{p-2}{p-3}\right)^{2} \prod_{\substack{p\left|P_{w, z} \\ p\right| \gamma}}\left(\frac{p-1}{p-2}\right)^{2}
$$

and $v(p)=0$ for $p \nmid \gamma, p<y$, or $p>z$, and

$$
\sum_{k \mid(a-\gamma)} u(k)=\prod_{\substack{y<p \leq w \\ p \mid(a-\gamma) \\ p \nmid \gamma}}\left(\frac{p-1}{p-3}\right)^{2} \prod_{\substack{w<p \leq z \\ p \mid(a-\gamma) \\ p \nmid \gamma}}\left(\frac{p}{p-2}\right)^{2}
$$

and $u(p)=0$ for $p \nmid(a-\gamma), p<y$, or $p>z$. Then, for any integer $k$,

$$
\left|v\left(p^{k}\right)\right| \leq\left\{\begin{array}{ll}
1 & \text { if } k=1, \\
0 & \text { if } k>1,
\end{array} \quad \text { and } \quad\left|u\left(p^{k}\right)\right| \leq \begin{cases}1 & \text { if } k=1 \\
0 & \text { if } k>1\end{cases}\right.
$$

Since Theorem 1 holds uniformly in $h$ and $\gamma$, the average we wish to estimate is

$$
\ll\left(\sum_{\gamma \leq x} \pi_{\gamma}(x)\right)^{-1}\left(\frac{1}{\sqrt{1+W(x)}} \sum_{\gamma \leq x} \Psi_{\gamma}(x) \Pi_{2}\right) .
$$

According to inequality (1), the first product may be estimated by

$$
\left(\frac{2 x}{\log ^{2} x} \sum_{\gamma \leq x} \prod_{\substack{p \mid \gamma \\ p>2}}\left(\frac{p-1}{p-2}\right)+O\left(\frac{x^{2}}{\log ^{c} x}\right)\right)^{-1} \ll \frac{\log ^{2} x}{x^{2}} .
$$

A direct calculation shows that

$$
\sum_{\gamma \leq x} \Psi_{\gamma}(x) \Pi_{2} \ll_{a} \frac{x}{\log ^{2} x}\left(x \sum_{k \leq|x-a|} \frac{u(k)}{k}\right)^{1 / 2}\left(x \sum_{d \leq x} \frac{v(d)}{d}\right)^{1 / 2} .
$$

Since $\sum_{d \leq x} v(d) / d \ll 1$ and $\sum_{k \leq x} u(k) / k \ll 1$, the above is not greater 
than $(x / \log x)^{2}$. As a result, we see that $(41)$ is

$$
\ll_{a} \frac{1}{\sqrt{1+W(x)}} .
$$

\section{References}

[1] E. Bombieri, Le grand crible dans la théorie analytique des nombres, Astérisque 18 (1974).

[2] P. D. T. A. Elliott, Probabilistic Number Theory, Vol. I, Springer, New York, 1979.

[3] - Multiplicative functions on arithmetic progressions. VI. More middle moduli, J. Number Theory 44 (1993), 178-208.

[4] - The concentration function of additive functions on shifted primes, Acta Math. 173 (1994), 1-35.

[5] H. Halberstam and H. E. Richert, Sieve Methods, Academic Press, London, 1974.

[6] H. Montgomery, Topics in Multiplicative Number Theory, Lecture Notes in Math. 227, Springer, Berlin, 1971.

[7] I. Z. Ruzsa, On the concentration of additive functions, Acta Math. Acad. Sci. Hungar. 36 (1980), 215-232.

[8] N. M. Timofeev, The Erdös-Kubilius conjecture concerning the value distribution of additive functions on the sequence of shifted primes, Acta Arith. 58 (1991), 113-131 (in Russian).

Department of Mathematical Sciences

Station \#18

Eastern New Mexico University

Portales, New Mexico 88130

U.S.A.

E-mail: wongs@math.enmu.edu

Received on 8.8.1995

and in revised form on 22.9.1997 